\title{
Complexity of PL manifolds
}

\author{
BRUnO MARTELLi
}

\begin{abstract}
We extend Matveev's complexity of 3-manifolds to PL compact manifolds of arbitrary dimension, and we study its properties. The complexity of a manifold is the minimum number of vertices in a simple spine. We study how this quantity changes under the most common topological operations (handle additions, finite coverings, drilling and surgery of spheres, products, connected sums) and its relations with some geometric invariants (Gromov norm, spherical volume, volume entropy, systolic constant).

Complexity distinguishes some homotopically equivalent manifolds and is positive on all closed aspherical manifolds (in particular, on manifolds with nonpositive sectional curvature). There are finitely many closed hyperbolic manifolds of any given complexity. On the other hand, there are many closed 4-manifolds of complexity zero (manifolds without 3-handles, doubles of 2-handlebodies, infinitely many exotic K3 surfaces, symplectic manifolds with arbitrary fundamental group).
\end{abstract}

57Q99; 57M99

\section{Introduction}

The complexity $c(M)$ of a compact 3-manifold $M$ (possibly with boundary) was defined in a nice paper of Matveev [29] as the minimum number of vertices of an almost simple spine of $M$. In that paper he proved the following properties:

Additivity $c\left(M \# M^{\prime}\right)=c(M)+c\left(M^{\prime}\right)$ for any (boundary-)connected sum.

Finiteness There are finitely many closed irreducible (or cusped hyperbolic) 3-manifolds of bounded complexity.

Monotonicity If $M_{S}$ is obtained by cutting $M$ along an incompressible surface $S$, then $c\left(M_{S}\right) \leqslant c(M)$.

Thanks to the combinatorial nature of spines, it is not hard to classify all manifolds having increasing complexity $0,1,2, \ldots$ Tables have been produced in various contexts; see Burton [8], Callahan, Hildebrand and Weeks [9], Frigerio, Martelli and Petronio [14], 
Martelli [25], Martelli and Petronio [26], Matveev [30; 31] and the references therein (and Table 1 below). Some of these classifications were actually done using the dual viewpoint of singular triangulations, which turns out to be equivalent to Matveev's for the most interesting 3-manifolds.

We extend here Matveev's complexity from dimension 3 to arbitrary dimension. To do this, we need to choose an appropriate notion of spine. In another paper [27] written in 1973, Matveev defined and studied simple spines of manifolds in arbitrary dimension. A simple spine of a compact manifold is a (locally flat) codimension-1 subpolyhedron with generic singularities, onto which the manifold collapses. If the manifold is closed there cannot be any collapse at all and we therefore need to priorly remove one ball.

Simple spines are actually not flexible enough for defining a good complexity. In dimension 3, as an example, any simple spine for $S^{3}$ (or, equivalently, $D^{3}$ ) is a complicated and unnatural object, such as Bing's house or the abalone. Every simple spine of $D^{3}$ has at least one vertex. However, a reasonable complexity must be zero on discs and spheres.

To gain more flexibility, Matveev defined in 1988 the more general class of almost simple spines [28; 29] of 3-manifolds. An almost simple polyhedron is a compact polyhedron that can be locally embedded in a simple one. This more general definition allows one to use very natural objects as spines, such as a point for $D^{3}$ or a circle for $D^{2} \times S^{1}$ : a point is not a vertex by definition, and hence $c\left(D^{3}\right)=0$, as required. We show here that the notion of almost simple spine extends naturally to all dimensions.

This is in fact not the only way to gain more flexibility. A different possibility consists in enlarging the notion of spine by admitting an arbitrary number of open balls in its complement. Following that road, a 2-sphere is a simple spine of $S^{3}$ (or $D^{3}$ ) without vertices, and hence $c\left(D^{3}\right)=0$ again. One might also allow simultaneously almost simple polyhedra and more balls in their complement.

In our attempt to define a suitable complexity in any dimension $n$, we are apparently forced to choose among three different definitions of complexity, and the choice seems only a matter of taste: as a spine for $S^{n}$, do we allow a point, an equator $(n-1)-$ sphere, or both?

Luckily, these three definitions are actually equivalent and lead to the same complexity $c\left(M^{n}\right)$, in every dimension $n$. This nontrivial fact shows that $c\left(M^{n}\right)$ is indeed a very natural quantity to associate to a compact manifold $M^{n}$. For the sake of clarity, we choose in Section 3 the simplest definition, which takes simple polyhedra and admits more balls in their complement. The other definitions and the proof of their equivalence are deferred to Section 7. 
Having settled the problem of defining $c$, we turn to studying its properties. Threedimensional complexity is already widely studied, and 1- and 2-dimensional ones are quite boring, so in this introduction we focus mainly on dimension 4 .

We start by studying how $c$ varies when a handle is added. Quite surprisingly, the complexity can always be controlled. If a 4 -manifold $N$ is obtained from $M$ by adding a handle of index $i>0$, we have $c(N) \leqslant c(M)$, except when $i=3$ : in that case we get the opposite inequality $c(N) \geqslant c(M)$. When $i=4$ we actually have $c(N)=c(M)$.

These simple inequalities already allow to prove many things, including that plenty of 4-manifolds have complexity zero, in contrast with the 3-dimensional case. These include all 4-manifolds (with or without boundary) having a handle-decomposition without 3-handles, and all the doubles of 2-handlebodies (ie manifolds decomposing without 3- and 4-handles). The first set includes many simply connected manifolds (maybe all), the second set contains closed manifolds with arbitrary (finitely presented) fundamental group.

We can find more. It is easy to see that a nontrivial product $M^{k} \times N^{n-k}$ with boundary has a spine without vertices. Therefore every closed 4-manifold obtained from a nontrivial product by adding handles of index $\neq 3$ has complexity zero. Among manifolds that may constructed in this way, we find the infinitely many exotic K3 surfaces discovered by Fintushel and Stern in [13] and the closed symplectic manifolds with arbitrary fundamental group exhibited by Gompf in [15] (both types of manifolds are built by attaching handles of index $\neq 3$ to a product $M^{3} \times S^{1}$ ).

As we have seen, there are plenty of 4-manifolds of complexity zero, although in many cases describing explicitly their spines is not obvious. One could guess that complexity is just zero on all 4 -manifolds. Luckily, this is not the case. Various nontriviality results (in all dimensions $n$ ) are proved in this paper.

A closed $n$-manifold $M$ with complexity zero must indeed fulfill some strict requirements. First of all, it cannot be aspherical. Moreover, its Gromov norm $\|M\|$ vanishes. If $\pi_{1}(M)$ is infinite and (virtually) torsion-free, some other geometric invariants of $M$ also vanish: the spherical volume $T(M)$ defined by Besson, Courtois and Gallot [7], the volume entropy $\lambda(M)$, and the systolic constant $\sigma(M)$, defined by Gromov [18].

Concerning Gromov norm, we actually have $c(M) \geqslant\|M\|$ for every closed aspherical manifold. This shows in particular that there are closed manifolds of arbitrarily high complexity in all dimensions. It also implies that there are finitely many closed hyperbolic $n$-manifolds of bounded complexity: this is a mild extension of the 3-dimensional finiteness property, proved by Matveev for all closed irreducible 3-manifolds. 
The triviality and nontriviality results just stated suggest that $c(M)$ is a well-balanced quantity which could reasonably measure how "complicate" a manifold is. We hope that this new invariant will help to understand better the enormous set of PL (equivalently, smooth) compact 4-manifolds.

Another kind of complexity for 4-manifolds (defined by Costantino and the author [10; 24]) makes use of 2-dimensional shadows instead of 3-dimensional spines: the two complexities are qualitatively different (see the end of Section 1).

\section{Structure of the paper}

In Section 1 we list all the properties of $c$ that are proved in this paper. Some basic notions of piecewise-linear topology are collected in Section 2. Simple spines and complexity are then introduced in Section 3. Some of our definitions are somehow different from the ones given by Matveev: in Sections 4 and 7 we prove that they are equivalent.

In Section 5 we construct simple spines as objects dual to triangulations. In Section 6 we show how to modify correspondingly a spine when the manifold is drilled along some subpolyhedron. This basic operation will be used at many stages in the rest of the paper.

In Section 8 we study how complexity changes under handle addition, sphere drilling, and connected sum. In Section 9 we study the complexity of products and of finite coverings. In Section 10 we introduce a generalization of normal surfaces to arbitrary dimension and show how to "cut" a simple spine along a normal hypersurface.

In Section 11 we study the nerve of a simple spine $P$ : the nerve is a simplicial complex determined by the stratification of $P$, which contains a lot of information on the topology of the manifold. The nerve is the key tool to prove various nontriviality results for $c$. The relations between complexity and homotopy invariants, Gromov norm, and Riemannian geometry are then studied in Sections 12, 13, and 14. Finally, Section 15 is devoted to four-manifolds.

Acknowledgements We would like to thank Katya Pervova and the anonymous referee for suggesting improvements on earlier versions of the manuscript. We also thank Roberto Frigerio and Roman Sauer for many helpful conversations on bounded cohomology and Gromov norm.

\section{Main results}

We define the complexity $c(M)$ of any compact PL manifold $M$ in Section 3 . The definition is of course also applicable to every smooth compact manifold by taking its 
unique PL structure (see Whitehead [39]). In this section we collect all the properties of $c$ proved in this paper.

\section{Topological operations}

Simple spines are flexible. Most topological operations on manifolds can be translated into some corresponding modifications of their spines. Various estimates on the complexity are therefore proved by examining how the number of vertices may vary along these modifications.

We collect here some estimates. We start with products.

Product with boundary $A$ product $N=M \times M^{\prime}$ with nonempty boundary has $c(N)=0$.

In other words, if either $M$ or $M^{\prime}$ is bounded, then $c\left(M \times M^{\prime}\right)=0$. If both $M$ and $M^{\prime}$ are closed, we may have $c\left(M \times M^{\prime}\right)>0$ : this holds for instance if both $M$ and $M^{\prime}$ are aspherical (and so $N$ is), for instance if $M=M^{\prime}=S^{1}$. On the other hand, we have the following.

Sphere product We have $c\left(M \times S^{n}\right)=0$ if $n \geqslant 2$.

Note that $\left\|M \times S^{n}\right\|=0$ for any $n \geqslant 1$. We are not aware of any general inequality relating $c(M), c\left(M^{\prime}\right)$, and $c\left(M \times M^{\prime}\right)$ when both manifolds are closed. We turn to coverings.

Covering If $\widetilde{M} \rightarrow M$ is a degree-d covering, then $c(\widetilde{M}) \leqslant d c(M)$.

In contrast with Gromov norm, this inequality is far from being an equality in general. For instance, lens spaces have arbitrarily high complexity while their universal covering $S^{3}$ has complexity zero.

We investigate the effect of adding a $i$-handle to a $n$-manifold $M$. Quite surprisingly, we always get a one-side estimate when $n \geqslant 4$, which depends only on the codimension $n-i$.

Handles Let $N$ be obtained from $M$ by adding a handle of index $i$. We have

- $c(N) \leqslant c(M)$ if $i<n-1$,

- $c(N) \geqslant c(M)$ if $i=n-1$ and $n \geqslant 4$,

- $c(N)=c(M)$ if $i=n$ and $n \geqslant 3$. 
These estimates imply a series of inequalities concerning connected sums and drilling along spheres of any dimension.

Connected sum We have $c\left(M^{n} \# N^{n}\right) \leqslant c\left(M^{n}\right)+c\left(N^{n}\right)$ for every (boundary) connected sum in dimension $n \geqslant 3$.

Matveev proved that an equality holds in dimension three [29]. We do not know if it still holds in dimension $n \geqslant 4$.

We turn to sphere drilling. If $S \subset M$ is a submanifold, we denote by $M_{S}$ the manifold obtained by removing from $M$ an open regular neighborhood of $S$. As for handle addition, if $S$ is a $k$-sphere and $n \geqslant 4$ we get a one-side estimate which depends only on the dimension $k$.

Sphere drilling Let $S \subset M$ be a $k$-sphere. We have

- $c\left(M_{S}\right) \leqslant c(M)$ if $k=1$ and $n \geqslant 4$,

- $c\left(M_{S}\right) \geqslant c(M)$ if $k>1$.

The (PL-)sphere $S$ does not need to be locally flat. If $S$ has a product regular neighborhood $D^{n-k} \times S$ we can perform a surgery by substituting this neighborhood with $S^{n-k-1} \times D^{k+1}$ along some map. If $k=1$, the previous result implies the following.

Surgery If $N$ is obtained from $M$ by surgery along a simple closed curve and $n \geqslant 4$, then $c(N) \leqslant c(M)$.

A strict inequality holds in some cases.

Strict inequality If $M$ is closed with $c(M)>0$ and $n \geqslant 4$, there is a simple closed curve $\gamma \subset M$ such that $c(N)<c(M)$ if $N$ is obtained by drilling or surgery along $\gamma$.

This implies the following result. Very often in dimension 4 a complicate manifold becomes "simpler" after summing it with $S^{2} \times S^{2}$ or $S^{2} \tilde{\times} S^{2} \cong \mathbb{C P}^{2} \# \overline{\mathbb{C P}}^{2}$. The complexity might estimate this phenomenon as follows.

Stabilization If $M$ is a simply connected closed 4-manifold with $c(M)>0$ then $c\left(M \#\left(S^{2} \times S^{2}\right)\right)<c(M)$ and $c\left(M \#\left(S^{2} \tilde{\times} S^{2}\right)\right)<c(M)$.

However, we do not know if there exists any simply connected 4-manifold of positive complexity! 
Finally, an important result of 3-dimensional complexity, due to Matveev [29], says that $c\left(M_{S}\right) \leqslant c(M)$ whenever $S$ is an incompressible surface. Unfortunately, the notion of incompressibility does not extend appropriately to higher dimensions. Having in mind that every class in $\mathrm{H}_{2}\left(M^{3}, \mathbb{Z}\right)$ is represented by an incompressible surface, we extend a weaker version of Matveev's result as follows.

Hypersurfaces Every class in $H_{n-1}\left(M^{n}, \mathbb{Z}_{2}\right)$ is represented by a hypersurface $S$ such that $c\left(M_{S}\right) \leqslant c(M)$.

This result is proved by extending the 3-dimensional notion of normal surface to any dimension: this extension might be of independent interest.

The results just stated are proved in Sections 8, 9 and 10.

\section{Gromov norm and triangulations}

Let $\|M\|$ and $t(M)$ be respectively the Gromov norm [17] and the minimum number of simplices in a triangulation of $M$.

Gromov norm (1) If $M$ is closed with virtually torsion-free $\pi_{1}(M)$, then

$$
\|M\| \leqslant c(M) \leqslant t(M) .
$$

Note that if $M$ is aspherical then $\pi_{1}(M)$ is torsion-free and hence the inequalities hold for any aspherical manifold $M$. Actually, only the left inequality requires this hypothesis on $\pi_{1}(M)$, and we do not know if it is really necessary. Both inequalities might be justified informally by saying that simples spines are more flexible than triangulations, but not as flexible as real homology cycles.

The above result can be strengthened in complexity zero, by dropping the hypothesis on $\pi_{1}(M)$ and admitting amenable boundary. The boundary $\partial M$ is amenable if the image of every connected component of $\partial M$ in $\pi_{1}(M)$ is an amenable group.

Gromov norm (2) Let $M$ be a manifold with (possibly empty) amenable boundary. If $c(M)=0$ then $\|M\|=0$.

The amenability hypothesis is necessary, since a genus- -2 handlebody has complexity zero and positive Gromov norm.

The results just stated are proved in Sections 5 and 13. 


\section{Homotopy type}

It might be that every simply connected manifold has complexity zero. This question is open only in dimension 4.

Simply connected Every simply connected compact manifold of dimension $\neq 4$ has complexity zero.

On the other hand, we have the following.

Arbitrary fundamental group Every finitely presented group is the fundamental group of a closed 4-manifold with complexity zero.

Complexity detects aspherical manifolds, in some sense.

Aspherical manifolds If $M$ is closed aspherical, then $c(M)>0$.

This shows in particular that complexity behaves quite differently from Gromov norm. For instance, complexity detects nonpositive curvature, while Gromov norm detects negative curvature: the $n$-torus $T^{n}$ has $c(T)>0$ and $\|T\|=0$.

We also note that complexity is not a homotopy invariant, since it distinguishes some homotopically equivalent lens spaces: we have $c\left(L_{7,1}\right)=4$ and $c\left(L_{7,2}\right)=2$ [29]. We do not know if it distinguishes different PL manifolds sharing the same topological structure.

The results just stated are proved in Section 12 .

\section{Riemannian geometry}

We compare the complexity of a smooth manifold $M$ with other invariants coming from Riemannian geometry. A relation between the volume of a Riemannian manifold and its complexity can be given by bounding both the sectional curvature and the injectivity radius. The second inequality in the following result is due to Gromov [17].

Volume Let $M^{n}$ be a Riemannian manifold with everywhere bounded sectional curvature $|K(M)| \leqslant 1$. Then

$$
c(M) \leqslant t(M) \leqslant \operatorname{const}_{n} \frac{\operatorname{Vol}(M)}{\operatorname{inj}_{*}(M)^{n}} .
$$


Here $\operatorname{inj}(M)$ is the injectivity radius, $\operatorname{inj}_{*}(M)=\min \{\operatorname{inj}(M), 1\}$, and const $_{n}$ is a constant depending on $n$. The same formula holds for Gromov norm $\|M\|:$ in that case however the factor $\operatorname{inj}_{*}(M)^{-n}$ can be removed when $\pi_{1}(M)$ is residually finite [17]. It is not possible to remove this factor here, since there are infinitely many hyperbolic 3manifolds with bounded volume, while only finitely many can have bounded complexity. This holds in fact in all dimensions.

Finiteness There are finitely many closed hyperbolic $n$-manifolds of bounded complexity, for every $n$.

We do not know if the finiteness property can be extended to manifolds of nonnegative curvature, or more generally to aspherical manifolds. As far as we know, it might also hold for elliptic manifolds. The results on Gromov norm allow to prove also the following.

Cusped hyperbolic manifolds Let $M$ be a compact manifold whose interior admits a complete hyperbolic metric of finite volume. Then $c(M)>0$.

This result is sharp since the Gieseking 3-manifold has complexity 1 [9]. Complexity is also related to other geometric invariants. A nice chain of inequalities, taken from [20;35], holds for every closed orientable manifold $M$ :

$$
\frac{n^{n / 2}}{n !}\|M\| \leqslant 2^{n} n^{n / 2} T(M) \leqslant \lambda(M)^{n} \leqslant h(M)^{n} \leqslant(n-1) \operatorname{MinVol}(M) .
$$

From left to right, we find Gromov norm $\|M\|$, the spherical volume $T(M)$ defined by Besson, Courtois and Gallot in [7], the volume entropy $\lambda(M)$, the topological entropy $h(M)$, and the minimum volume $\operatorname{Min} \operatorname{Vol}(M)$ defined by Gromov in [17]. Another interesting invariant is the systolic constant $\sigma(M)$, defined by Gromov in [18].

Geometric invariants Let $M$ be a closed orientable manifold with virtually torsionfree infinite fundamental group. If $c(M)=0$ then

$$
T(M)=\lambda(M)=\sigma(M)=0 .
$$

We do not know if the same hypothesis implies also $h(M)=0$. It does not imply $\operatorname{MinVol}(M)=0$ (for instance, if $M=\left(T^{2} \times S^{2}\right) \# \mathbb{C P}^{2}$ we have $c(M)=0$ and $\operatorname{Min} \operatorname{Vol}(M) \geqslant$ const $\cdot|\chi(M)|>0[17])$.

Finally, we quote a result of Alexander and Bishop [2; 3] relating the complexity and the width of a Riemannian manifold with boundary. 
Thin manifolds There are some constants $a_{2}<a_{3}<\cdots$ such that if a Riemannian manifold $M^{n}$ with boundary has (curvature-normalized) inradius less than $a_{n}$, then $c\left(M^{n}\right)=0$.

The results just stated are proved in Section 14 .

\section{Low dimensions}

The complexity of manifolds of dimension 1 and 2 is easily calculated. Concerning 1 -manifolds, we have $c\left(S^{1}\right)=c\left(D^{1}\right)=1$. Turning to dimension 2 , the complexity of a (compact) surface $\Sigma$ turns out to be as follows:

- $c(\Sigma)=\max \{2-2 \chi(\Sigma), 0\}$ if $\Sigma$ is closed.

- $c(\Sigma)=\max \{-2 \chi(\Sigma), 0\}$ if $\Sigma$ has boundary.

The compact surfaces having complexity zero are $S^{2}, \mathbb{R} \mathbb{P}^{2}, D^{2}$, the annulus and the Möbius strip. The torus and the pair-of-pants have complexity 2 .

The complexity of 3-manifolds has been widely studied. Manifolds of low complexity have been listed via computer by various authors $[8 ; 9 ; 14 ; 25 ; 26 ; 30 ; 31]$ : the closed orientable irreducible ones are collected in Table 1 according to their geometry. The closed irreducible manifolds having complexity zero are $S^{3}, \mathbb{R P}^{3}$ and $L_{3,1}$.

\begin{tabular}{|c|c|c|c|c|c|c|c|c|c|c|c|c|}
\hline$c$ & 0 & 1 & 2 & 3 & 4 & 5 & 6 & 7 & 8 & 9 & 10 & 11 \\
\hline lens spaces & 3 & 2 & 3 & 6 & 10 & 20 & 36 & 72 & 136 & 272 & 528 & 1056 \\
\hline other elliptic & . & . & 1 & 1 & 4 & 11 & 25 & 45 & 78 & 142 & 270 & 526 \\
\hline flat & . & . & . & . & . & . & 6 & . & $\cdot$ & $\cdot$ & $\cdot$ & $\cdot$ \\
\hline Nil & . & . & . & . & . & . & 7 & 10 & 14 & 15 & 15 & 15 \\
\hline $\mathrm{SL}_{2} \mathbb{R}$ & . & . & . & . & . & . & . & 39 & 162 & 513 & 1416 & 3696 \\
\hline Sol & . & . & . & . & . & . & . & 5 & 9 & 23 & 39 & 83 \\
\hline $\mathbb{H}^{2} \times \mathbb{R}$ & . & . & . & . & . & . & . & . & 2 & $\cdot$ & 8 & 4 \\
\hline hyperbolic & . & . & . & . & . & . & . & . & . & 4 & 25 & 120 \\
\hline not geometric & . & . & . & . & . & . & . & 4 & 35 & 185 & 777 & 2921 \\
\hline & & 2 & 4 & 1 & 17 & 31 & 74 & 175 & 436 & 1154 & 3078 & 8421 \\
\hline
\end{tabular}

Table 1: The number of irreducible orientable 3-manifolds of complexity $c \leqslant 11$ in each geometry. The nongeometric manifolds decompose into geometric pieces according to their JSJ decomposition along tori (they are all graph manifolds when $c \leqslant 10$ ). 
We now devote our attention to dimension 4 . We start by studying the set of 4manifolds of complexity zero. We describe here some interesting classes of such manifolds. These classes seem however far to exhaust the set of all 4-manifolds with complexity zero.

The various results stated above show that the set of all 4-manifolds of complexity zero contains all products $N \times N^{\prime}$ with nonempty boundary or $N \in\left\{S^{2}, S^{3}\right\}$, and is closed under connected sums, finite coverings, addition of handles of index $\neq 3$, and drilling (or surgery) along simple closed curves. All the examples presented here are of this kind. We concentrate on closed manifolds for simplicity.

No 3-handles Every closed 4-manifold that has a handle decomposition without 3-handles has complexity zero.

Every such manifold is necessarily simply connected. However, for many simply connected manifolds a decomposition without 3-handles does not seem to be known. Among these, we find the exotic K3 surfaces constructed by Fintushel and Stern in [13]. In fact, these manifolds are constructed by attaching handles of index $\neq 3$ to a product $M^{3} \times S^{1}$. Therefore we have the following.

Exotic K3 The (infinitely many) exotic K3 surfaces $X_{K}$ constructed via Fintushel and Stern's knot construction [13] from a knot $K \subset S^{3}$ have complexity zero.

We now introduce two different classes of closed 4-manifolds with arbitrary (finitely presented) fundamental group. Let a 2-handlebody be a 4-manifold which has a decomposition with $0-, 1-$ and 2-handles.

Doubles of 2-handlebodies The double of any orientable 2-handlebody has complexity zero.

These manifolds have complexity zero since they are obtained by surgering $\left(S^{1} \times S^{3}\right)$ \# $\ldots \#\left(S^{1} \times S^{3}\right)$ along some curves. Every finitely presented group is the fundamental group of a 2-handlebody, which is in turn isomorphic to the fundamental group of its double. It is not true that any double has complexity zero, because a double can be aspherical (for instance, a product of surfaces).

Another class was constructed by Gompf in [15], in order to show that symplectic 4-manifolds may have arbitrary fundamental group. As above, these manifolds are constructed by attaching handles of index $\neq 3$ to a product $M^{3} \times S^{1}$, so we have the following. 
Symplectic manifolds The closed symplectic manifolds with arbitrary fundamental group constructed by Gompf in [15] have complexity zero.

The results just stated are proved in Section 15.

\section{Relation with shadow-complexity}

There is another natural way to extend Matveev's complexity to dimension 4: instead of taking 3-dimensional spines, one may take 2-dimensional shadows. ${ }^{1}$ A shadowcomplexity was introduced by Costantino [10] and the author [24]. The resulting function $c^{\text {shadow }}$ is quite different from the complexity $c^{\text {spine }}=c$ defined here.

The closed 4-manifolds having shadow-complexity zero were described in [23]. A closed orientable $M^{4}$ has $c^{\text {shadow }}\left(M^{4}\right)=0$ if and only if $M=N^{4} \#_{h} \mathbb{C P}^{2}$ for some "graph manifold" $N^{4}$ and some $h \in \mathbb{Z}$. These graph manifolds are doubles of 2-handlebodies (of a particular simple form), and thus we get the following:

Shadow-complexity If $c^{\text {shadow }}\left(M^{4}\right)=0$ then $c^{\text {spine }}\left(M^{4}\right)=0$.

The converse does not hold: as shown in [23], the only closed simply connected 4manifolds $M$ with $c^{\text {shadow }}(M)=0$ are $S^{4}, \mathbb{C P}^{2}, S^{2} \times S^{2}$ and their connected sums, so in particular we have $c^{\text {shadow }}(K 3)>0$.

\section{Piecewise-linear topology}

We collect here the information on piecewise-linear topology that we will need. The basic definitions and tools are listed in Section 2.1 and Section 2.2. More material can be found in Rourke and Sanderson [37]. The notion of intrinsic stratification is taken from Akin [1], Armstrong [4], McCrory [32] and Stone [38] and described in Section 2.3. Stein factorization (which we take from Costantino and Thurston [11]) is introduced in Section 2.4. Finally, in Section 2.5 we define the nerve of a pair $(X, Y)$ of polyhedra: this definition is original and might be of independent interest.

The material contained in Sections 2.4 and 2.5 is used only in Section 11 to define the nerve of a pair $(M, P)$ when $P$ is a simple spine of $M$.

\footnotetext{
${ }^{1}$ As defined by Turaev, a shadow $P^{2} \subset M^{4}$ is a simple polyhedron such that $M^{4}$ is a regular neighborhood of $P$ plus some 3 - and 4 -handles.
} 


\subsection{Basic definitions}

2.1.1 Simplicial complexes A (finite and abstract) simplicial complex $K$ is a set of nonempty subsets of a given finite set $V(K)$ (the vertices of $K$ ), such that $\{v\} \in K$ for all $v \in V(K)$ and if $\sigma \in K$ and $\tau \subset \sigma$ then $\tau \in K$. An element of $K$ is a face. A subcomplex is a subset of $K$ which is a complex. If $K$ and $L$ are simplicial complexes, a simplicial map $f: K \rightarrow L$ is a function $f: V(K) \rightarrow V(L)$ such that if $\sigma \in K$ then $f(\sigma) \in L$.

2.1.2 Triangulations A (finite) simplicial complex $K$ induces a compact topological space $|K|$, defined by taking a standard Euclidean simplex for each element of $K$ and identifying them according to the face relations. A triangulation of a compact topological space $X$ is a simplicial complex $K$ and a homeomorphism $f:|K| \rightarrow X$. Another triangulation $(L, g)$ of $X$ is a subdivision of $(K, f)$ if the image of every simplex of $L$ is contained as a straight simplex in some simplex of $K$. Two triangulations of $X$ are related if they have a common subdivision.

We will use the letter $T$ to indicate a triangulation, ie a pair $(K, f)$.

2.1.3 Polyhedra A compact polyhedron is a compact topological space $X$ equipped with a maximal family of related triangulations. A subpolyhedron $X^{\prime} \subset X$ is a subset which is the image of a subcomplex of some triangulation of $X$. If $X$ is a polyhedron containing compact polyhedra $X_{1}, \ldots, X_{k}$, a triangulation $K$ of $\left(X, X_{1}, \ldots, X_{k}\right)$ is a triangulation of $X$ where each $X_{i}$ is represented by some subcomplex; such a triangulation can be found by taking a common subdivision of the triangulations realizing $X_{i}$ as a subcomplex.

The standard $n$-simplex $\Delta^{n}$ is a polyhedron. We define the $n$-disc $D^{n}$ and $(n-1)-$ sphere $S^{n-1}$ respectively as $\Delta^{n}$ and $\partial \Delta^{n}$.

2.1.4 Manifolds and maps A simplicial map $f: K \rightarrow L$ induces a continuous map $f:|K| \rightarrow|L|$. A map between polyhedra is piecewise-linear (shortly, PL) if it is induced by a simplicial map on some triangulations. A polyhedron is a PL-manifold (with boundary) if it is locally PL-homeomorphic to some point in $S^{n}\left(D^{n}\right)$. Every manifold and map mentioned in this paper is tacitly assumed to be PL.

\subsection{Basic tools}

2.2.1 Derived complexes A simplicial complex $K$ defines a partially ordered set (briefly, a poset) $i(K)=(K, \subseteq)$, the set of faces with their face relations. Conversely, a poset $(A, \leqslant)$ defines a simplicial complex $\eta(A, \leqslant)$, whose vertices are the elements 
of $A$, and whose faces are all finite subsets $\left\{a_{0}, \ldots, a_{i}\right\}$ such that $a_{0}<\cdots<a_{i}$. The simplicial complex $\eta(A, \leqslant)$ is the nerve of $(A, \leqslant)$.

The simplicial complex $K^{\prime}=\eta \circ i(K)$ is the derived simplicial complex of $K$. Vertices of $K^{\prime}$ correspond to faces of $K$. A simplicial map $f: K \rightarrow L$ induces an orderpreserving map $i(K) \rightarrow i(L)$ and hence a derived simplicial map $f^{\prime}: K^{\prime} \rightarrow L^{\prime}$.

A triangulation $T=(K, f)$ of a space $X$ determines a barycentric subdivision $T^{\prime}=$ $\left(K^{\prime}, f^{\prime}\right)$ of $X$, obtained by composing $f$ with the homeomorphism $\left|K^{\prime}\right| \rightarrow|K|$ which sends every vertex of $K^{\prime}$ to the barycenter of the corresponding face of $K$ (and is extended linearly on the rest of $\left|K^{\prime}\right|$ ).

2.2.2 Join, cone and suspension The join $K * L$ of two simplicial complexes $K$ and $L$ (with disjoint vertices) is the complex with vertices $V(K * L)=V(K) \cup V(L)$ and with faces $K \cup L \cup\{\sigma \cup \tau \mid \sigma \in K, \tau \in L\}$. The polyhedron $|K * L|$ depends only on $|K|$ and $|L|$ (up to homeomorphism) and can thus be denoted by $|K| *|L|$.

The cone and suspension of a polyhedron $P$ are respectively $C(P)=P * D^{0}$ and $\Sigma(P)=P * S^{0}$. We have $\Sigma^{k}(P) \cong P * S^{k-1}$.

2.2.3 Link, star and regular neighborhood Let $K$ be a simplicial complex and $L \subset K$ a subcomplex. The star $\operatorname{st}(L, K)$ of $L$ in $K$ is the minimal subcomplex of $K$ containing all faces that intersect some face of $L$. The $\operatorname{link} \operatorname{lk}(L, K)$ is the subcomplex of $\operatorname{st}(L, K)$ consisting of all faces not intersecting any face of $L$.

When $Y \subset X$ are polyhedra and $T$ is a triangulation of $(X, Y)$, we indicate by $\operatorname{lk}(Y, T)$ and $\operatorname{st}(Y, T)$ the corresponding subpolyhedra of $X$. When $Y=\{y\}$ is a point, these polyhedra depend (up to homeomorphism) only on $y$ and not on $T$.

In general, if $T$ is sufficiently subdivided, the $\operatorname{star} \operatorname{st}(Y, T)$ does not depend on $T$ up to an isotopy in $X$ keeping $Y$ fixed: for instance, this holds after two barycentric subdivisions. In that case, the polyhedron $\operatorname{st}(Y, T)$ is the regular neighborhood of $Y$ in $X$, which we denote by $R(Y)$.

When $X$ is a manifold, the regular neighborhood $R(Y)$ is a manifold with boundary.

2.2.4 Collapse Let $K$ be a simplicial complex. Let $\sigma \in K$ be a face which is properly contained in a unique face $\eta$. The subcomplex $L=K \backslash\{\sigma, \eta\}$ is obtained from $L$ by an elementary collapse.

Let $Y \subset X$ be any polyhedra. The polyhedron $Y$ is obtained from $X$ via a elementary collapse if it is so on some triangulation. More generally, a collapse of $X$ onto a subpolyhedron $Z$ is a combination of finitely many simplicial collapses. 


\subsection{Intrinsic strata}

We recall the notions of intrinsic dimension and strata of polyhedra; see Akin [1], Armstrong [4], McCrory [32] and Stone [38].

Let $Y \subset X$ be any polyhedra and $x \in X$ a point. The intrinsic dimension $d(x ; X, Y)$ of the pair $(X, Y)$ at $x$ is the maximum number $t$ such that the following holds:

(1) There is a triangulation of $(X, Y)$ with $x$ contained in the interior of a $t$-simplex.

If $x \in Y$, this is equivalent to each of the following conditions:

(2) The link of $x$ in $(X, Y)$ is the $t$-th suspension $\Sigma^{t}(W, Z)$ of some pair $(W, Z)$.

(3) The star of $x$ in $(X, Y)$ is homeomorphic to $C(W, Z) \times D^{t}$ with $x$ sent to $v \times c$, where $v$ is the vertex of the cone $C(W, Z)$ and $c \in \operatorname{int}\left(D^{t}\right)$.

The absolute notion of intrinsic dimension of a point $x$ in a polyhedron $Y$ is defined as $d(x ; Y)=d(x ; Y, Y)$. If $x \notin Y$ we have $d(x ; X, Y)=d(x ; X)$. If $x \in Y$ we have $d(x ; X, Y) \leqslant\{d(x, X), d(x, Y)\}$.

A subpolyhedron $Y \subset X$ in a manifold $X$ is locally unknotted at $x$ if $d(x ; X, Y)=$ $d(x ; Y)$. When $Y$ is a manifold, this is equivalent to the standard notion of local flatness. The subpolyhedron $Y \subset X$ is locally unknotted if it is so at every $x \in Y$.

The intrinsic dimension can be easily calculated using the following nice result of Armstrong [4] and Morton [34]:

Proposition 2.1 (Armstrong-Morton) If the link of $x$ in $(X, Y)$ is the $t$-th suspension of some pair $(W, Z)$, and $(W, Z)$ is not itself a suspension, then $t=d(x ; X, Y)$ and $(W, Z)$ is uniquely determined by $x$.

This easily implies the following.

Exercise 2.2 If $Y \subset X$ is locally unknotted, then $\operatorname{lk}(x, Y) \subset \operatorname{lk}(x, X)$ is locally unknotted for every $x \in Y$.

The intrinsic dimension induces an intrinsic stratification of any pair $(X, Y)$. The points of intrinsic dimension $k$ in $Y$ form the $k$-stratum of $Y$. The $k$-stratum is an (open) $k$-dimensional manifold made of finitely many connected components, called $k$-components (or simply components). Points in a $k$-component are all homogeneous, ie there is an ambient isotopy of $Y$ sending a point to any other. In particular, they have the same link.

The union of all points of intrinsic dimension $\leqslant k$ is the $k$-skeleton: it is a $k-$ dimensional polyhedron. 


\subsection{Stein factorization}

A Stein factorization of a (piecewise-linear) map $f: X \rightarrow Y$ between (compact) polyhedra is a decomposition $f=g \circ h$ into two maps

$$
X \stackrel{h}{\longrightarrow} Z \stackrel{g}{\longrightarrow} Y
$$

such that $h$ has connected fibers and $g$ is finite-to-one. Every $f$ has a unique Stein factorization: the map $h$ is the quotient onto the space $Z$ of connected components of the fibers of $f$; see Figure 1. We learned about this notion from [11].

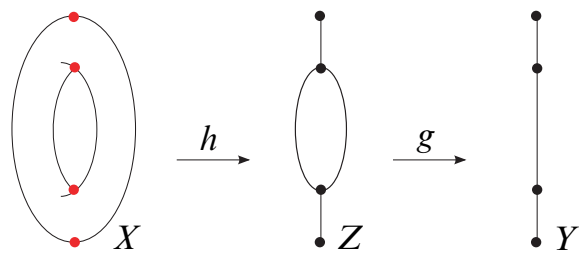

Figure 1: The Stein factorization of a map

We define the Stein factorization in the category of simplicial complexes. Let $f: K \rightarrow L$ be a simplicial map. Let $f^{\prime}: K^{\prime} \rightarrow L^{\prime}$ be its derived map. We define an intermediate simplicial complex $H$ as follows. Consider the map $f^{\prime}:\left|K^{\prime}\right| \rightarrow\left|L^{\prime}\right|$. The vertices of $H$ are the connected components of $\left(f^{\prime}\right)^{-1}(v)$ when $v$ varies among the vertices of $L^{\prime}$. The map $f^{\prime}: V\left(K^{\prime}\right) \rightarrow V\left(L^{\prime}\right)$ naturally splits along two maps

$$
V\left(K^{\prime}\right) \stackrel{h}{\longrightarrow} V(H) \stackrel{g}{\longrightarrow} V\left(L^{\prime}\right) .
$$

We now define a simplex in $H$ to be the image of any simplex in $K^{\prime}$ along $h$. The resulting maps

$$
K^{\prime} \stackrel{h}{\longrightarrow} H \stackrel{g}{\longrightarrow} L^{\prime}
$$

are simplicial and $f^{\prime}=g \circ h$. Since we used the derived map $f^{\prime}$, the map $h:\left|K^{\prime}\right| \rightarrow|H|$ has indeed connected fibers everywhere (not only at the vertices of $H$ ). The map $g:|H| \rightarrow\left|L^{\prime}\right|$ is finite-to-one: this is equivalent to the condition that $\operatorname{dim} g(\sigma)=\operatorname{dim} \sigma$ for every simplex $\sigma$ of $H$.

\subsection{Nerve}

The nerve of a polyhedron is a simplicial complex which encodes the incidences between its components; see Section 2.3. We define it for pairs $(X, Y)$.

Let $Y \subset X$ be any polyhedra. The components of $(X, Y)$ form a partially ordered set $(\mathcal{C}, \leqslant)$ : we set $C \leqslant C^{\prime}$ if $C \subset \overline{C^{\prime}}$. If $C<C^{\prime}$ then $\operatorname{dim} C<\operatorname{dim} C^{\prime}$. The prenerve of $(X, Y)$ is the nerve $\mathcal{N}_{0}=\eta(\mathcal{C}, \leqslant)$ of this partially ordered set; see Section 2.2.1 above. 
Let $T$ be a triangulation of $(X, Y)$. If $T$ is sufficiently subdivided, by sending every vertex of $T$ to the component to which it belongs we get a surjective simplicial map $\varphi_{0}: T \rightarrow \mathcal{N}_{0}$, called the prenerve map. It induces a surjective continuous map $\varphi_{0}: X \rightarrow\left|\mathcal{N}_{0}\right|$.

The prenerve map does not necessarily have connected fibers, so we prefer to consider its Stein factorization; see Section 2.4. The nerve of $(X, Y)$ is the complex $\mathcal{N}$ obtained via the Stein factorization

$$
T^{\prime} \stackrel{\varphi}{\longrightarrow} \mathcal{N} \stackrel{g}{\longrightarrow} \mathcal{N}_{0}^{\prime}
$$

of the prenerve map $\varphi_{0}^{\prime}=g \circ \varphi$. The map $\varphi: T^{\prime} \rightarrow \mathcal{N}$ is the nerve map. More generally, a nerve map is a map $\varphi: X \rightarrow|\mathcal{N}|$ induced by some (sufficiently subdivided) triangulation of $(X, Y)$.

Exercise 2.3 The prenerve of $(X, Y)=\left(S^{1},\{\mathrm{pt}\}\right)$ is a segment, while the nerve is a circle.

\section{Simple polyhedra}

The definition of simple polyhedra in arbitrary dimensions is due to Matveev [27]. We use it in Section 3.3 to define the complexity of a $n$-manifold. This definition extends Matveev's complexity of 3-manifolds [29].

\subsection{The local model}

Let $\Delta=\Delta^{n+1}$ be the $(n+1)$-simplex. Let $\Pi^{n}$ be the cone over the $(n-1)$-skeleton of $\Delta$. The base of the cone is its boundary $\partial \Pi^{n}$, while $\operatorname{int}\left(\Pi^{n}\right)=\Pi^{n} \backslash \partial \Pi^{n}$ is its interior. Some examples are shown in Figure 2.

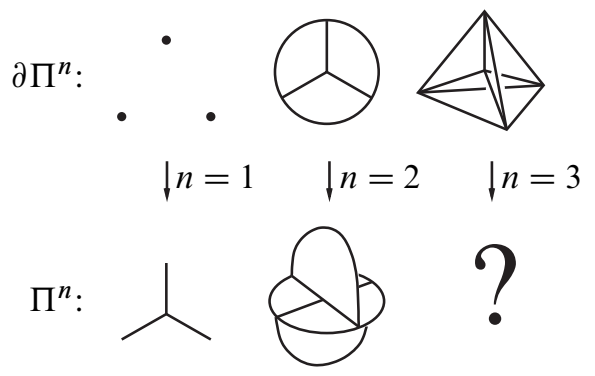

Figure 2: The $(n-1)$-skeleton of the $(n+1)$-simplex and the cone $\Pi^{n}$ over it. The three-dimensional $\Pi^{3}$ is not drawn. 

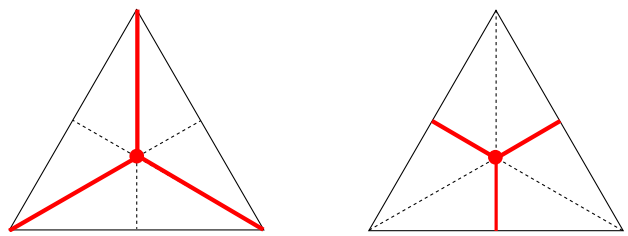

Figure 3: The standard and dual representation of $\Pi^{n}$ inside $\Delta$. They are both subcomplexes of $\Delta^{\prime}$. Here, $n=1$.

There are two representations of $\Pi^{n}$ inside $\Delta$, shown in Figure 3: the standard and dual representation. They both describe $\Pi^{n}$ as a subcomplex of the barycentric subdivision $\Delta^{\prime}$. See also Figure 4. Both representations induce the same pair $\left(D^{n+1}, \Pi^{n}\right)$

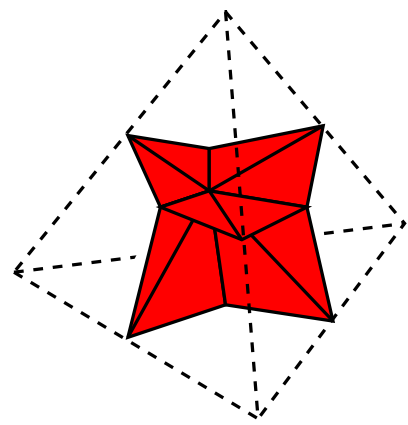

Figure 4: The dual representation of $\Pi^{2}$ inside the tetrahedron $\Delta^{3}$

up to homeomorphism. The dual representation is investigated below in Section 5.1. We define $\Pi_{k}^{n}$ as $\Pi_{k}^{n}=\Pi^{n-k} \times D^{k}$. The pair $\left(D^{n+1}, \Pi_{k}^{n}\right)=D^{k} \times\left(D^{n-k+1}, \Pi^{n-k}\right)$ is well-defined up to homeomorphism. The boundary $\partial \Pi_{k}^{n}=\Pi_{k}^{n} \cap S^{n}$ is homeomorphic to the $k$-th suspension $\Sigma^{k}\left(\partial \Pi^{n-k}\right)$. Following Matveev, a point $x$ in a polyhedron $P$ is of type $k$ if its link is homeomorphic to $\partial \Pi_{k}^{n}$ (and hence its star is homeomorphic to $\left.\Pi_{k}^{n}\right)^{2}$. See Figure 5.

The polyhedron $\Pi^{n}$ has a natural triangulation induced by that of $\Delta$.

Proposition 3.1 A point $x \in \operatorname{int}\left(\Pi^{n}\right)$ has intrinsic dimension $k$ if and only if it is of type $k$.

Proof Since $\partial \Pi^{n-k}$ is not a suspension, a point of type $k$ has intrinsic dimension $k$ by Proposition 2.1.

${ }^{2}$ Actually, our $\Pi_{k}^{n}$ corresponds to Matveev's $\Pi_{n-k}^{n}$ : we prefer to define the type of a point coherently with Armstrong's general notion of intrinsic dimension. 

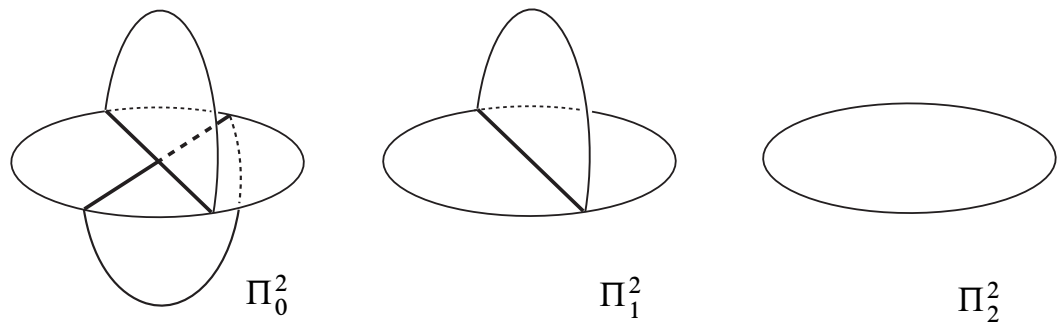

$\Pi_{1}^{2}$

$\Pi_{2}^{2}$

Figure 5: The local models of a simple polyhedron of dimension 2

The polyhedron $\Pi^{n}$ may be constructed recursively. In the following, we see both $\Pi^{n-1}$ and $S^{n-1}$ inside $D^{n}$. See Figure 6.

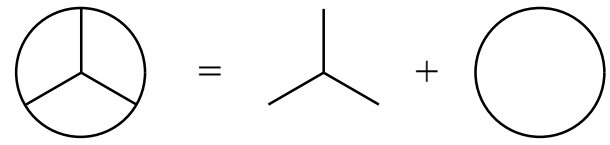

Figure 6: We have $\partial \Pi^{n} \cong \Pi^{n-1} \cup S^{n-1}$. Here $n=2$.

Proposition 3.2 We have $\partial \Pi^{n} \cong \Pi^{n-1} \cup S^{n-1}$.

Proof Take a vertex $v$ and the opposite face $f$ in $\Delta^{n+1}$. The $(n-1)$-skeleton of $\Delta^{n+1}$ is the union of $\partial f$ and a cone over the $(n-2)$-skeleton of $f$ with base $v$. $\square$

Corollary 3.3 We have $\Pi^{n} \cong\left(\Pi^{n-1} \times[0,1]\right) \cup\left(D^{n} \times\{0\}\right)$.

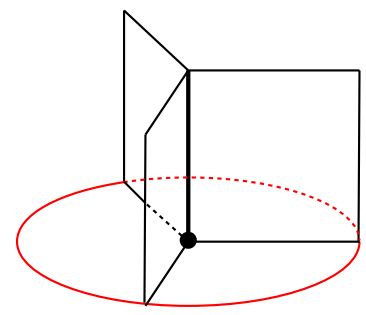

Figure 7: We have $\Pi^{n} \cong\left(\Pi^{n-1} \times[0,1]\right) \cup\left(D^{n} \times\{0\}\right)$. Here $n=2$ : the disc $D^{2} \times\{0\}$ is horizontal and $\Pi^{1} \times[0,1]$ is vertical.

See Figure 7. The following is an easy corollary of Proposition 2.1.

Exercise 3.4 If $X$ is a polyhedron such that $X \times[-1,1]^{h} \cong \Pi_{k}^{n}$ then $X \cong \Pi_{k-h}^{n}$. 


\subsection{Simple polyhedron}

Definition 3.5 A compact polyhedron $P^{n}$ is simple if every point of $P$ is of some type $k$ (that is, its link is homeomorphic to $\partial \Pi_{k}^{n}$ ).

See some examples in Figure 8. A point of type 0 is called a vertex. In this paper, $n=1$ :
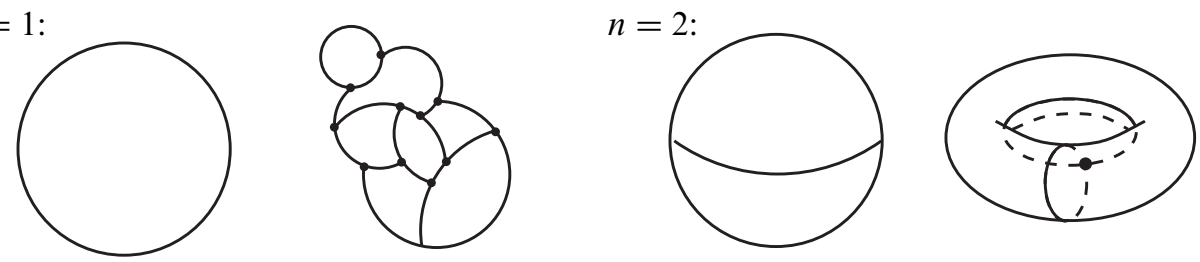

Figure 8: Simple polyhedra of dimension $n=1$ (a circle and a trivalent graph) and $n=2$ (a sphere and a torus with two discs attached)

every simple polyhedron $P \subset \operatorname{int}\left(M^{n}\right)$ contained in some manifold $M^{n}$ will be tacitly assumed to have codimension 1 and to be locally unknotted; see Section 2.3. This is equivalent to require that $P$ is properly embedded in Matveev's sense [27]: the equivalence is proved in Section 4.1. Local unknottedness is actually automatic in dimension $n \leqslant 4$; see Remark 4.3 below.

Exercise 3.6 The polyhedron $\partial \Pi^{n}$ is simple with $n+2$ vertices.

The exercise is also proved as Corollary 5.7 below.

\subsection{Complexity}

A spine of a manifold is usually defined as a subpolyhedron onto which the manifold collapses. This definition however applies only to manifolds with boundary: in order to extend it to closed manifolds, we allow the removal of an arbitrary number of open balls.

Definition 3.7 Let $M$ be a compact manifold. A subpolyhedron $P \subset \operatorname{int}(M)$ is a spine of $M$ if there are some disjoint discs $D_{1}, \ldots, D_{k} \subset \operatorname{int}(M)$, disjoint also from $P$, such that $M \backslash \operatorname{int}\left(D_{1} \cup \cdots \cup D_{k}\right)$ collapses onto $P$.

See some examples in Figure 9. We are now ready to define the complexity of a manifold.

Definition 3.8 The complexity $c(M)$ of a compact manifold $M$ is the minimum number of vertices in a simple spine of $M$. 

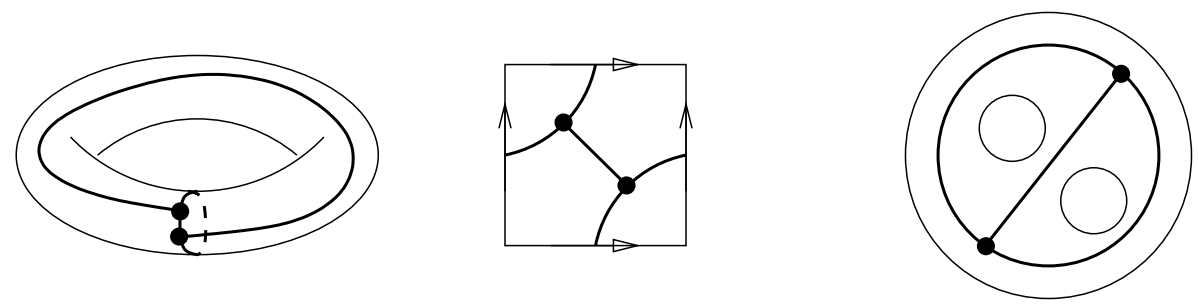

Figure 9: A spine of the torus (left and center) and of the pair-of-pants (right)

Every compact manifold admits a simple spine (see Matveev [27] or Corollary 5.5 below) and hence this quantity is indeed finite. A simple spine $P \subset M$ is minimal if it has $c(M)$ vertices.

\subsection{Examples}

The equator $(n-1)$-sphere is a simple spine of $S^{n}$ : the $n$-sphere collapses to it after removing two small balls centered at the poles. Analogously, a hyperplane is a simple spine of $\mathbb{R} \mathbb{P}^{n}$ (the manifold $\mathbb{R P}^{n}$ collapses to it after removing one ball). When $n \geqslant 2$ these spines have no vertices and therefore $c\left(S^{n}\right)=c\left(\mathbb{R P}^{n}\right)=0$. When $n=1$, the circle $S^{1}$ has a point as a simple spine, which is indeed a vertex, and hence $c\left(S^{1}\right)=1$.

Figure 9 shows a spine with 2 vertices of the 2 -torus $T$ : hence $c(T) \leqslant 2$. It is easy to see that $T$ has no spine with lower number of vertices, and hence $c(T)=2$. A similar argument shows the following.

Exercise 3.9 The complexity $c(\Sigma)$ of a closed surface $\Sigma$ is

- $c(\Sigma)=\max \{2-2 \chi(\Sigma), 0\}$ if $\Sigma$ is closed,

- $c(\Sigma)=\max \{-2 \chi(\Sigma), 0\}$ if $\Sigma$ has boundary.

The surfaces having complexity zero are $S^{2}, \mathbb{R P}^{2}$, the annulus, and the Möbius strip. They all have a circle as a spine without vertices.

Many examples in dimension 3 can be found in the literature (see Burton [8], Martelli and Petronio [26], Martelli [25] and Matveev [29; 31]), so we turn to higher-dimensional manifolds. A nice spine for $\mathbb{C P}^{n}$ can be described by using a technique which was inspired to us by tropical geometry as in Mikhalkin [33]. Consider the projection

$$
\begin{aligned}
p: \mathbb{C P}^{n} & \longrightarrow \Delta^{n} \\
{\left[z_{0}, \ldots, z_{n}\right] } & \longmapsto\left(\frac{\left|z_{0}\right|}{\left|z_{0}\right|+\cdots+\left|z_{n}\right|}, \ldots, \frac{\left|z_{n}\right|}{\left|z_{0}\right|+\cdots+\left|z_{n}\right|}\right) .
\end{aligned}
$$


Consider $\Pi^{n-1}$ dually embedded in $\Delta^{n}$. The counterimage $p^{-1}\left(\Pi^{n-1}\right)$ is a simple spine of $\mathbb{C P}^{n}$ without vertices (its complement consists of $n+1$ open balls "centered" at the points $[0, \ldots, 0,1,0, \ldots, 0])$. Therefore $c\left(\mathbb{C P}^{n}\right)=0$.

The spine of $\mathbb{C P}^{2}$ fibers over $\Pi^{1}$. It consists of three solid tori attached to one 2-torus. We find such a spine also from a different construction. Let $M^{4}$ be a closed 4-manifold which decomposes with $0-, 2-$ and 4-handles only. The attaching of the 2-handles is encoded by a framed link $L \subset S^{3}$. Let $P$ be the union of the boundaries of all the handles involved. It consists of a 3 -sphere $S^{3}$ plus one solid torus attached to (a regular neighborhood of) each component of $L$. When $L$ is the $1-$ framed unknot we find $M^{4}=\mathbb{C P}^{2}$ and we get the same spine as above. In general, we get a simple spine of $M$ without vertices (all points are of type 3 or 2). Therefore $c\left(M^{4}\right)=0$.

\section{Collars}

As proved by Matveev [27], a locally unknotted simple polyhedron $P \subset M$ has a kind of collar, similar to a collar of the boundary of a manifold. We introduce the collar by defining the cut map in Section 4.2. To do this, we first need to prove that Matveev's notion of local flatness (which is more useful in the context of simple spines) coincides with the general one introduced in Section 2.3.

\subsection{Matveev's definition}

Matveev introduced in [27] a different definition of local unknottedness for simple polyhedra, which is more useful here. We show that it coincides with the general one introduced in Section 2.3. The proof is not strictly necessary (we could use Matveev's notion and forget about the general one), but we include it for completeness.

We defined the pair $\left(D^{n}, \Pi_{k}^{n-1}\right)$ in Section 3.1. The following definition is due to Matveev [27].

Definition 4.1 A simple polyhedron $P^{n-1} \subset M^{n}$ in a manifold $M^{n}$ is properly embedded if the link of every point in $(M, P)$ is homeomorphic to $\left(S^{n-1}, \partial \Pi_{k}^{n-1}\right)$ for some $k$.

Proposition 4.2 A simple polyhedron $P \subset \operatorname{int}(M)$ of codimension 1 is locally unknotted if and only if it is properly embedded.

Proof It is easy to see that a properly embedded $P^{n-1} \subset M^{n}$ is locally unknotted. We prove the converse by induction on $n$. The case $n=1$ is trivial, so we assume $n \geqslant 2$. 
Let $x$ be a point of $P$, of some type $k$. The link of $x$ in $(M, P)$ is homeomorphic to $\left(S^{n-1}, Y^{n-2}\right)$ with $Y^{n-2} \cong \partial \Pi_{k}^{n-1}$. We must show that the homeomorphism extend to pairs, ie that $\left(S^{n-1}, Y^{n-2}\right) \cong\left(S^{n-1}, \partial \Pi_{k}^{n-1}\right)$.

Since $P$ is locally unknotted, the pair $\left(S^{n-1}, Y^{n-2}\right)$ is also locally unknotted by Exercise 2.2. The polyhedron $Y$ is simple by Exercise 3.6, and is hence properly embedded by our induction hypothesis. Since $Y \cong \partial \Pi_{k}^{n-1}$ is a special polyhedron (ie a simple polyhedron whose $(n-2)$-components are discs), [27, Theorem 3] ensures that the homeomorphism $Y \cong \partial \Pi_{k}^{n-1}$ indeed extends to a regular neighborhood and hence to the whole of $S^{n-1}$, as required.

Remark 4.3 Local unknottedness is automatic in dimension $n \leqslant 4$ : in these dimensions, every embedding of $\partial \Pi_{k}^{n-1}$ in $S^{n-1}$ is in fact easily seen to be standard. In dimension 5, a nonstandard pair $\left(S^{4}, S^{3}\right)$, if it exists, could lead to nonstandard embeddings of $\Pi_{k}^{3}$ in $S^{4}$.

\subsection{Cut map}

As noted by Matveev [27], the locally unknotted embedding of a simple polyhedron allows us to define a collar, similar to the collar of a boundary in a manifold.

Let $P \subset \operatorname{int}(M)$ be a simple polyhedron in a compact manifold. By cutting $M$ along $P$ as suggested in Figure 10, we get a manifold $M_{P}$ with boundary and a surjective map $f: M_{P} \rightarrow M$.
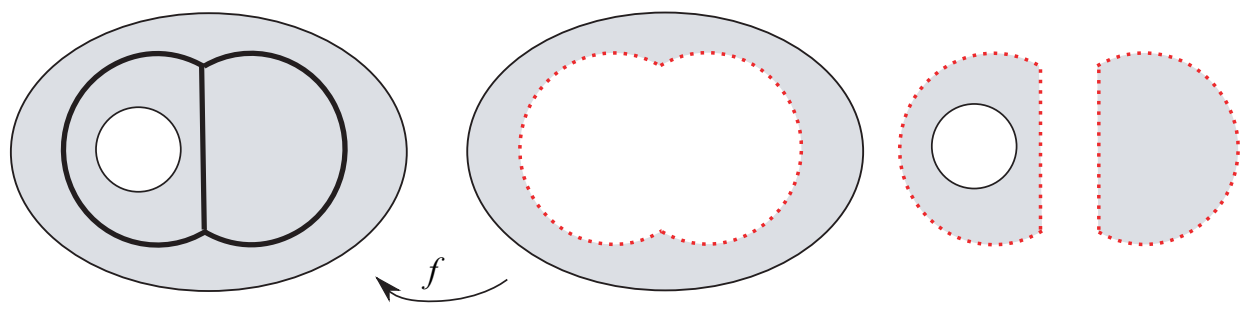

Figure 10: The cut map $f: M_{P} \rightarrow M$ cuts the manifold $M$ along the simple polyhedron $P$. The dotted boundary is $\partial_{0} M_{P}$.

The set $f^{-1}(P) \subset M_{P}$ consists of some components of $\partial M_{P}$, which we denote by $\partial_{0} M_{P}$. The map $f$ is a local embedding. It is $(n-k+1)$-to- 1 over a point of type $k$ in $(M, P)$. In particular, it restricts to a homeomorphism of $M_{P} \backslash \partial_{0} M_{P}$ onto $M \backslash P$. Regular neighborhoods $R(P)$ of $P$ in $M$ correspond via $f$ to collars of $\partial_{0} M_{P}$. The function $f$, restricted to one such collar, gives a collar $\partial R(P) \times[0,1] \rightarrow R(P)$ of $P$, as shown in [27]. This discussion implies in particular the following. 
Proposition 4.4 Let $P \subset \operatorname{int}(M)$ be a simple polyhedron. It is a spine of $M$ if and only if

$$
M_{P}=N \times[0,1] \sqcup D_{1} \sqcup \cdots \sqcup D_{k}
$$

where $D_{1}, \ldots, D_{k}$ are discs, $N$ is a possibly disconnected $(n-1)-$ manifold, and $\partial_{0} M_{P}=N \times 0$.

In other words, a simple polyhedron $P \subset \operatorname{int}(M)$ is a spine if and only if $M_{P}$ consists of a collar and some discs.

\section{Triangulations}

We describe here a construction which builds a simple polyhedron $P \subset M$ from a triangulation of $M$ and a partition of its vertices. From this we will deduce that $c(M) \leqslant t(M)$ for any compact $M$.

\subsection{Dual models}

We generalize the dual representation of $\Pi^{n}$ inside the simplex $\Delta=\Delta^{n+1}$ to the polyhedra $\Pi_{k}^{n}$. Let $\mathcal{P}=\left\{V_{0}, \ldots, V_{k}\right\}$ be a partition of the set $V$ of vertices of $\Delta$. Every $V_{i}$ spans a face $f_{i}$ of $\Delta$.

Definition 5.1 The polyhedron dual to $(\Delta, \mathcal{P})$ is

$$
Z=\bigcup_{i=0}^{k} \operatorname{lk}\left(f_{i}, \Delta^{\prime}\right) \text {. }
$$

When $\mathcal{P}=\{V\}$ we have $Z=\varnothing$. For the other cases, we have the following.

Proposition 5.2 We have $Z \cong \Pi_{n+1-k}^{n}$.

Proof If each $f_{i}$ consists of one vertex we get the dual representation of $\Pi^{n}$ and we are done. Otherwise, let $\Delta^{k}$ be a $k$-dimensional simplex, with vertices $w_{0}, \ldots, w_{k}$. By sending $V_{i}$ to $w_{i}$ we get a simplicial map $\phi: \Delta^{n+1} \rightarrow \Delta^{k}$. This induces another simplicial map $\phi^{\prime}:\left(\Delta^{n+1}\right)^{\prime} \rightarrow\left(\Delta^{k}\right)^{\prime}$ between the derived complexes. Let $Z_{*}$ be the polyhedron dual to $\left(\Delta^{k},\left\{w_{0}, \ldots, w_{k}\right\}\right)$. We have $f^{-1}\left(Z_{*}\right)=Z$ and $Z_{*} \cong \Pi^{k}$.

On a small neighborhood of the center $C$ of $\left(\Delta^{n+1}\right)^{\prime}$ the map $f^{\prime}$ is isomorphic to the projection $D^{k} \times D^{n-k+1} \rightarrow D^{k}$. Therefore the star of $C$ in $Z$ is homeomorphic to $\Pi^{k-1} \times D^{n-k+1} \cong \Pi_{n-k+1}^{n}$. Such a star is homeomorphic to $Z$ and we are done. $\square$

See a couple of examples in Figure 11. 


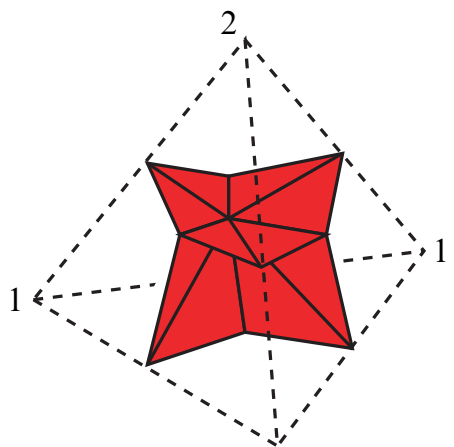

3

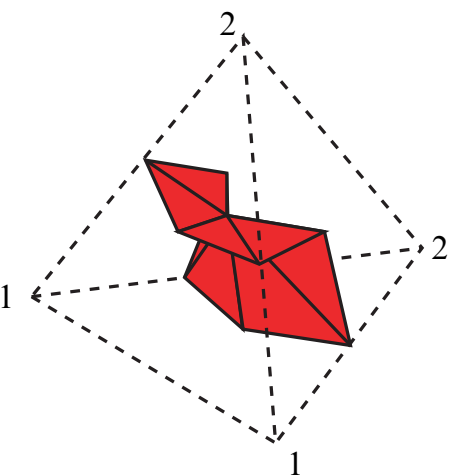

1

Figure 11: Models dual to some partitions of the vertices. Here we find $\Pi_{1}^{2}$ and $\Pi_{2}^{2}$.

\subsection{Simple polyhedra dual to triangulations}

Let $M$ be a closed manifold and $T$ a triangulation of $M$. Let $\mathcal{P}$ be a partition of the vertices of $T$. In each simplex $\sigma$ of $T$ we have an induced partition of its vertices and hence a dual polyhedron $P_{\sigma} \subset \sigma$.

Definition 5.3 The polyhedron dual to $(T, \mathcal{P})$ is

$$
P=\bigcup_{\sigma \in T} P_{\sigma}
$$

The dual polyhedron is a subcomplex of the barycentric subdivision $T^{\prime}$. For every set $V \in \mathcal{P}$ of the partition, we define the submanifold $M_{V} \subset M$ as the regular neighborhood in $T^{\prime \prime}$ of the union of all simplices in $T$ whose vertices lie in $V$. See Figure 12.
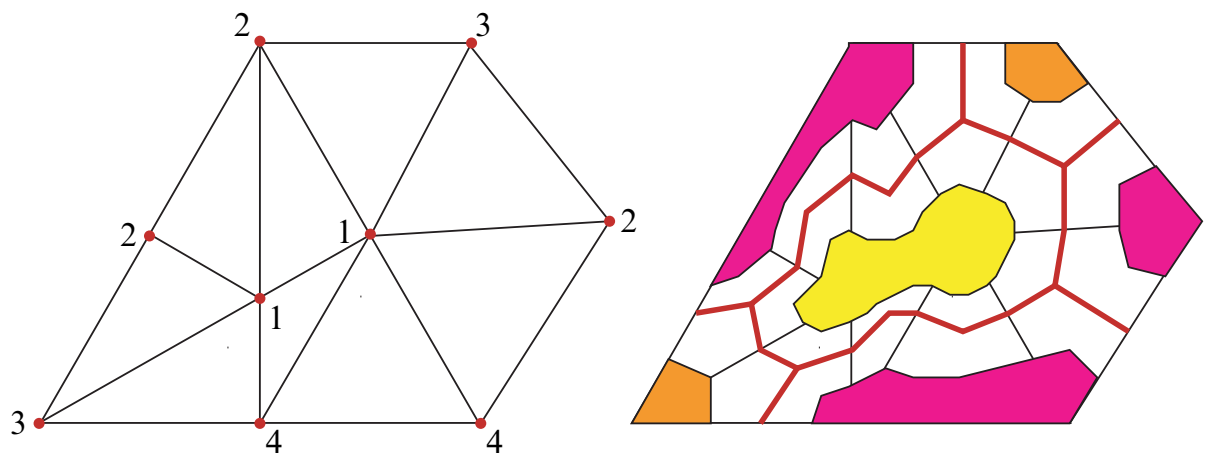

Figure 12: A triangulation $T$ and a partition of the vertices induce a simple polyhedron realized as a subcomplex of $T^{\prime}$ and some submanifolds $M_{V}$ realized as subcomplexes of $T^{\prime \prime}$. 
Proposition 5.4 Let $\mathcal{P}$ be a partition and $P$ be the polyhedron dual to $(T, \mathcal{P})$. Then $P$ is simple and the following holds.

(1) The vertices of $P$ are the barycenters of the simplices in $T$ whose vertices lie in $n+1$ distinct sets of $\mathcal{P}$.

(2) The regular neighborhood of $P$ in $T^{\prime \prime}$ is $M \backslash \operatorname{int}\left(\bigcup_{V \in \mathcal{P}} M_{V}\right)$.

Proof The proof is straightforward.

Let $t(M)$ be the minimum number of simplices arising in a triangulation of $M$.

Corollary 5.5 We have $c(M) \leqslant t(M)$ for every closed $M$.

Proof Let $T$ be a triangulation with $t=t(M)$ simplices. Let $\mathcal{P}$ be the discrete partition: distinct vertices belong to distinct sets. The polyhedron dual to $(T, \mathcal{P})$ is a spine of $M$ by Proposition 5.4 (2), since $M_{V}$ consists of discs (stars of the inner vertices). It has $t$ vertices by Proposition 5.4 (1).

Remark 5.6 The term "triangulation" is sometimes used for short in dimensions 2 and 3 to indicate a singular triangulation, ie the realization of a manifold $M^{n}$ as the union of some $n$-simplices whose faces are identified in pairs via some simplicial maps. This is not the case here: in this paper we employ the word "triangulation" only in its original PL meaning.

\subsection{Simple subpolyhedra of $\partial \Pi^{n}$}

Proposition 5.4 yields the following.

Corollary 5.7 The polyhedron $\partial \Pi_{k}^{n}$ is simple.

Proof Represent $\Pi_{k}^{n}$ as the dual of some partition $(\Delta, \mathcal{P})$. The boundary is the simple polyhedron dual to the same partition $(\partial \Delta, \mathcal{P})$.

Proposition 5.2 shows that $\partial \Pi_{k}^{n}$ is homeomorphic to some simple subpolyhedron of $\partial \Pi^{n}$. Conversely, we have the following result (which will be used in Section 7).

Proposition 5.8 See $\partial \Pi^{n}$ dually contained in $\partial \Delta$. If $n \geqslant 2$, every simple subpolyhedron of $\partial \Pi^{n}$ is dual to some partition $\mathcal{P}$ of the vertices of $\Delta$ and is hence homeomorphic to $\partial \Pi_{k}^{n}$ for some $k$. 
Proof Let $X$ be a simple subpolyhedron of $\partial \Pi^{n}$. A simple subpolyhedron of a simple polyhedron of the same dimension $n-1$ is necessarily the closure of the union of some $(n-1)$-components.

The $(n-1)$-components of $\partial \Pi^{n}$ are dual to the edges of $\Delta$. We define an equivalence relation on the vertices of $\Delta$ as follows: $v \sim v^{\prime}$ if and only if the $(n-1)$-component dual to the edge $v v^{\prime}$ is not contained in $X$. We check the transitive property: let $v, v^{\prime}, v^{\prime \prime}$ be vertices with $v \sim v^{\prime}$ and $v^{\prime} \sim v^{\prime \prime}$, and suppose that $v \not v^{\prime \prime}$. The triangle $v v^{\prime} v^{\prime \prime}$ is thus dual to a $(n-2)$ component which is adjacent to a unique $(n-1)-$ component contained in $X$ : this is impossible since $X$ is simple (and does not contain points with link homeomorphic to $D^{n-2}$ ).

The polyhedron $X$ is dual to the partition induced by this equivalence relation.

\section{Drilling}

Generic soap bubbles in $\mathbb{R}^{3}$ form a simple polyhedron. Moreover, if a new bubble appears generically somewhere, the polyhedron remains simple. This fact can be generalized to any dimension, as follows.

Let $Q \subset \operatorname{int}(M)$ be a simple polyhedron in a manifold. Let $K \subset \operatorname{int}(M)$ be any compact subpolyhedron. The operation of drilling $Q$ along $K$ consists of removing from $Q$ a small regular neighborhood of $K$ and adding its boundary as in Figures 13 and 14. More precisely, let $T$ be a triangulation of $(M, K, Q)$. Let $R=R\left(K, T^{\prime \prime}\right)$ be the regular neighborhood of $K$ in the twice subdivided $T^{\prime \prime}$. The result of this operation is the polyhedron

$$
P=(Q \backslash R) \cup \partial R
$$
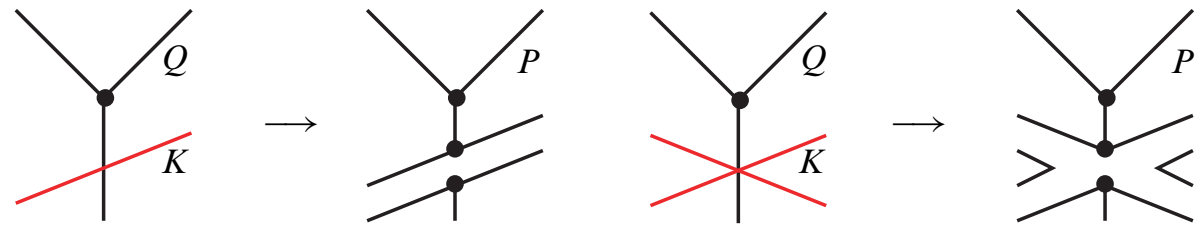

Figure 13: The simple polyhedron $P$ is obtained from $Q$ by drilling along $K$

Lemma 6.1 The polyhedron $P$ is simple. If $K$ does not intersect the 1 -skeleton of $Q$, then $P$ has the same vertices as $Q$. 

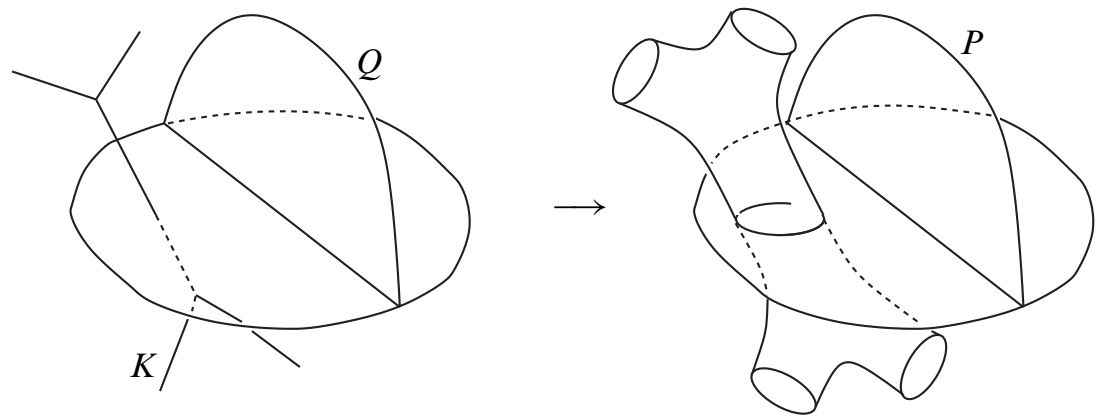

Figure 14: The simple polyhedron $P$ is obtained from $Q$ by drilling along $K$

Proof We have $P=(Q \backslash R) \cup \partial R$. We have to check that every point $x \in P$ is of some type $k$. If $x \in Q \backslash \partial R$ we are done because $Q$ is simple. Suppose $x \in \partial R$. Let $k \geqslant 1$ be the type of $x$ in $(M, Q)$. We show that $x$ is of type $k-1$ in $(M, P):$ in particular, $P$ is simple.

If $k=n$, ie $x \notin Q$, then $x$ is of type $(n-1)$ in $P$ because $\partial R$ is a $(n-1)-$ manifold. If $k<n$, the polyhedra $\partial R$ and $Q$ intersect transversely at $x$ (in the sense of Armstrong [4]). See an example in Figure 15: locally, $\partial R$ is a horizontal disc $D^{n-1} \times 0$ and $Q$ is a vertical product $Y \times[-1,1]$. Exercise 3.4 implies that $Y \cong \Pi_{k-1}^{n-1}$.
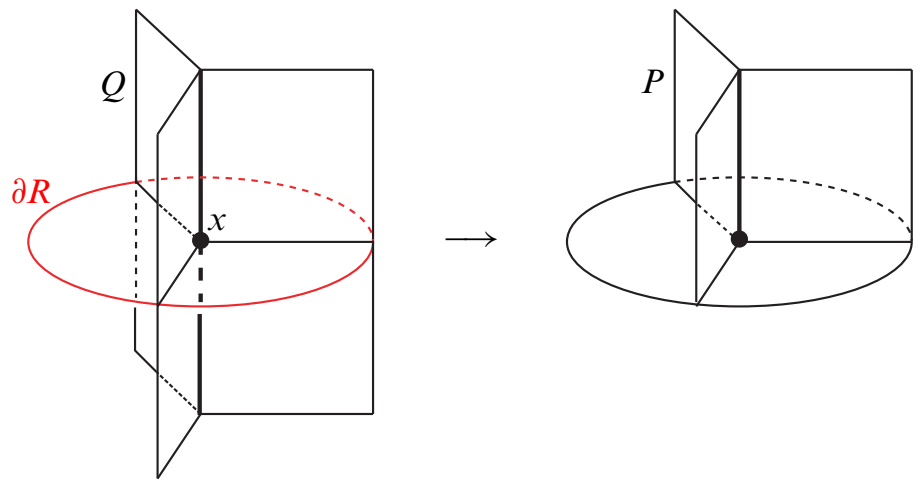

Figure 15: At $x$ the polyhedra $Q$ and $\partial R$ intersect transversely, so $\partial R$ cuts $Q$ into two halves. Then $P$ is obtained by discarding the half of $Q$ lying inside $R$ and adding $\partial R$.

The star of $x$ in $P$ is thus homeomorphic to

$$
\left(\Pi_{k-1}^{n-1} \times[0,1]\right) \cup\left(D^{n} \times 0\right) \cong D^{k-1} \times\left(\left(\Pi^{n-k} \times[0,1]\right) \cup\left(D^{n-k+1} \times 0\right)\right) .
$$


By Corollary 3.3, this is homeomorphic to $D^{k-1} \times \Pi^{n-k+1} \cong \Pi_{k-1}^{n}$. Therefore $x$ is of type $k-1$ in $P$.

Finally note that if $K$ does not intersect the $1-$ skeleton of $Q$ neither does $R$. Therefore every point in $Q \cap \partial R$ is of type $k>1$ in $Q$, and hence of type $k-1>0$ in $P$ : no new vertices are added to $Q$.

\section{Alternative definitions}

Matveev's original definition of complexity $c\left(M^{3}\right)$ for a 3-manifold $M^{3}$ was slightly different from ours. We prove here that the two definitions coincide.

A couple of natural variations might be done in our definition of complexity. The definition of "simple polyhedron" can be weakened by allowing the presence of lowerdimensional material. This choice is natural, since it allows to consider a point as a spine of $D^{n}$ or $S^{n}$. Matveev called such polyhedra almost simple. On the other hand, the definition of "spine" can be strengthened, by allowing the removal of one ball only when strictly necessary, ie when the manifold is closed. We call this more restricted notion a strict spine.

We therefore get $2 \times 2=4$ possible definitions of the complexity of a manifold. Luckily, it turns out that three of them coincide. These include ours and Matveev's definition (in dimension 3).

\subsection{Almost simple polyhedra}

Matveev employed in dimension 3 a more relaxed notion of polyhedron, called almost simple [29]. We propose the following generalization to all dimensions.

Definition 7.1 Let $M^{n}$ be a compact manifold. A compact subpolyhedron $P \subset$ $\operatorname{int}(M)$ is almost simple if the link of every point in $(M, P)$ is homeomorphic to $\left(S^{n-1}, L\right)$ for some subpolyhedron $L \subset \partial \Pi^{n-1} \subset S^{n-1}$.

See an example in Figure 16. A vertex of $P$ is a point whose link is homeomorphic to $\left(S^{n-1}, \partial \Pi^{n-1}\right)$. We now define $c^{\mathrm{alm}}(M)$ as the minimum number of vertices of an almost simple spine of $M$.

Example 7.2 A point $\{\mathrm{pt}\} \subset S^{n}$ is an almost simple spine of the $n$-sphere. Note that a point is not a vertex when $n \geqslant 2$, and hence $c^{\text {alm }}\left(S^{n}\right)=0$ for all $n \geqslant 2$. A hyperplane $H \subset \mathbb{C P}^{n}$ is an almost simple spine of complex projective space without 


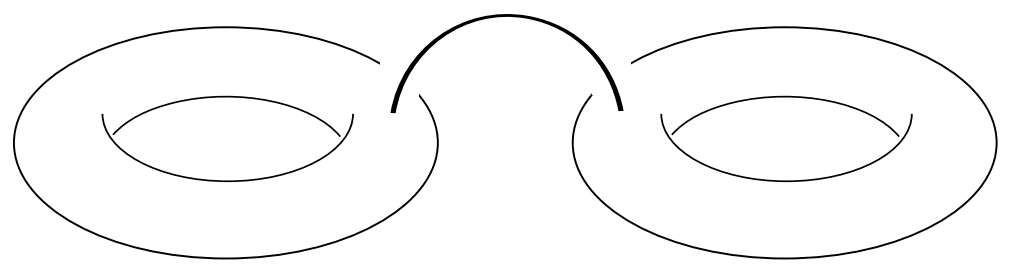

Figure 16: An almost simple spine of the boundary-connected sum $\left(T^{2} \times D^{1}\right) \#\left(T^{2} \times D^{1}\right)$. It consists of two tori joined by an arc. It has no vertices.

vertices, and hence $c^{\mathrm{alm}}\left(\mathbb{C P}^{n}\right)=0$. This spine is not simple (it has codimension 2): note that the construction of a simple spine of $\mathbb{C P}^{n}$ without vertices is less immediate; see Section 3.4.

We show below that $c=c^{\text {alm }}$ : to prove this, we need a couple of preliminary lemmas, which show how to construct a simple spine from an almost simple one without increasing the number of vertices. This is done first by collapsing (Lemma 7.3) and then by drilling along a triangulation of the low-dimensional part $K$ of the spine (Lemma 7.4).

Lemma 7.3 Every almost simple polyhedron $P \subset \operatorname{int}\left(M^{n}\right)$ collapses onto $Q \cup K$ where $Q$ is simple and $\operatorname{dim} K<n-1$. Every vertex of $Q$ is also a vertex of $P$.

Proof We prove this by induction on $n=\operatorname{dim} M$. If $n=2$ it is easy, so we turn to the case $n \geqslant 3$. Take a triangulation of $(M, P)$ and collapse $P$ as more as possible. The resulting polyhedron is some $Q \cup K \subset P$, where $Q$ (resp. $K$ ) is the closure of the set of all points whose link has dimension $n-2$ (resp. $<n-2)$.

We now prove that $Q$ is simple. Since $P$ is almost simple, the link of $x$ in $(M, Q, K)$ is homeomorphic to $\left(S^{n-1}, Q^{\prime}, K^{\prime}\right)$ for some $Q^{\prime} \cup K^{\prime} \subset \partial \Pi^{n} \subset S^{n-1}$. The polyhedron $Q^{\prime} \cup K^{\prime}$ cannot be collapsed onto a proper subpolyhedron, because $(Q, K)$ cannot. By our induction hypothesis $Q^{\prime}$ is hence simple. Proposition 5.8 implies that $Q^{\prime} \cong \partial \Pi_{k}^{n-2}$ and hence $x$ is of type $k$ in $Q$.

Lemma 7.4 If $M^{n}$ has a spine $Q \cup K \subset \operatorname{int}\left(M^{n}\right)$ such that $Q$ is simple with $t$ vertices and $\operatorname{dim} K<n-1$, then $c\left(M^{n}\right) \leqslant t$.

Proof Take a triangulation $T$ of $(M, Q, K)$. Let $\sigma_{1}, \ldots, \sigma_{k}$ be the simplices of $K$ that are not contained in $Q$, and not contained in any higher-dimensional simplex of $K$. We have $Q \cup K=Q \cup \sigma_{1} \cup \cdots \cup \sigma_{k}$. 
Each $\sigma_{i}$ is a cell (ie a disc) of dimension $<n-1$. We want to drill inductively along each $\sigma_{i}$. Since each $\sigma_{i}$ is a cell, each drilling produces a new open ball in the complement, so the final simple polyhedron is a spine. In order to not create new vertices, at each step we put the cells in general position. See Figure 17.
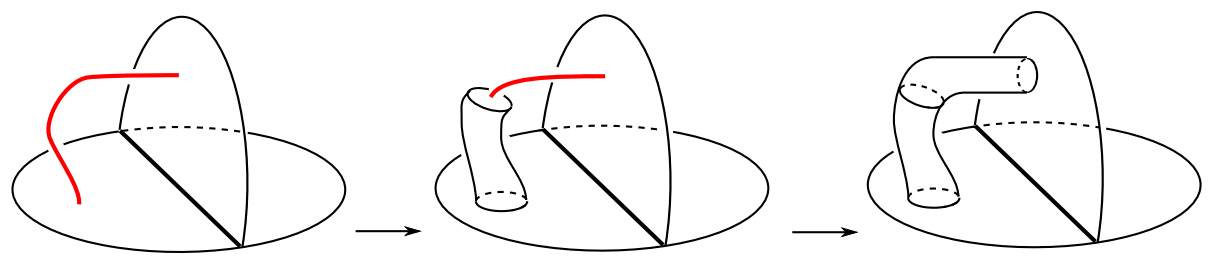

Figure 17: Let $Q \cup K$ be a spine of $M$, such that $Q$ is simple and $\operatorname{dim} K<$ $\operatorname{dim} Q$. We obtain a simple spine of $M$ by subdividing $K$ into cells and then drilling them inductively. Each drilling produces a new ball in the complement. Here, $K$ is an arc subdivided into two 1 -cells.

More precisely, we construct for each $i=0, \ldots, k$ a simple polyhedron $Q_{i}$ with $t$ vertices and some cells $\sigma_{1}^{i}, \ldots, \sigma_{k-i}^{i}$ of dimension $<n-1$ intersecting themselves and $Q_{i}$ only at their boundaries, such that $Q_{i} \cup \sigma_{1}^{i} \cup \cdots \cup \sigma_{k-i}^{i}$ is a spine of $M^{n}$. For $i=0$, take $Q_{0}=Q$ and $\sigma_{j}^{0}=\sigma_{j}$.

Let now $Q_{i}$ and $\sigma_{1}^{i}, \ldots, \sigma_{k-i}^{i}$ be defined for some $i<k$. Since $\operatorname{dim} \sigma_{j}^{i}<n-1$, we can perturb the cells so that they do not intersect the 1 -skeleton of $Q$. To do this, we use a collar of $P$ (see Section 4.2): we lift the cells to $M_{Q_{i}}$, perturb them slightly, and project them back. The perturbed polyhedron is still a spine.

Let $Q_{i+1}$ be obtained from $Q_{i}$ by drilling along $\sigma_{k-i}^{i}$. The polyhedron $Q_{i+1}$ is simple with $t$ vertices by Lemma 6.1. To drill we use a triangulation $T$ of the whole data $\left(M, Q_{i}, \sigma_{1}^{i}, \ldots, \sigma_{k-i}^{i}\right)$ : this ensures that $\sigma_{j}^{i+1}=\overline{\sigma_{j}^{i} \backslash Q_{i+1}}$ is still a cell.

Finally, $Q_{k}$ is a simple spine of $M$ with $t$ vertices.

Theorem 7.5 We have $c^{\text {alm }}(M)=c(M)$ for every compact manifold $M$.

Proof A simple spine is almost simple, hence $c^{\text {alm }}(M) \leqslant c(M)$. Conversely, an almost simple spine $P$ of $M$ with $c^{\text {alm }}(M)$ vertices collapses by Lemma 7.3 onto a $Q \cup K$ such that $Q$ has at most $c^{\mathrm{alm}}(M)$ vertices, and hence $c(M) \leqslant c^{\mathrm{alm}}(M)$ by Lemma 7.4. 


\subsection{Strict spines}

Matveev's notion of "spine" was more rigid than ours.

Definition 7.6 Let a strict spine $P \subset \operatorname{int}(M)$ of a compact manifold $M$ with boundary be a compact $P$ onto which $M$ collapses. A strict spine of a closed $M$ is defined as a strict spine of $M \backslash \operatorname{int}(D)$ for some disc $D^{n} \subset M^{n}$.

In contrast with spines, the complement of a strict spine contains one ball only if strictly necessary. Let $c_{\mathrm{str}}^{\mathrm{alm}}(M)$ be the minimum number of vertices of an almost simple strict spine. Matveev's original definition of the complexity of a 3-manifold $M^{3}$ is precisely $c_{\mathrm{str}}^{\mathrm{alm}}\left(M^{3}\right)$. We can finally show that his definition coincides with ours. The following result actually holds in all dimensions.

Theorem 7.7 We have $c_{\mathrm{str}}^{\mathrm{alm}}(M)=c(M)$ for every compact manifold $M$.

Proof Since a strict spine is a spine, we have $c_{\mathrm{str}}^{\mathrm{alm}}(M) \geqslant c^{\mathrm{alm}}(M)$. We now show the converse. Let $P$ be a minimal almost simple spine, ie with $c^{\text {alm }}(M)$ vertices. We now construct a strict one without increasing the number of vertices.

If $P$ is not strict, the complement $M \backslash P$ has some redundant balls: for each such $B$, there must be a $(n-1)$-component of $P$ which is adjacent to $B$ on one side and to another component of $M \backslash P$ on the other (because $M$ is connected). By removing a small open $(n-1)$-ball from this $(n-1)$-component we get an almost simple spine with one ball less in its complement. After finitely many such removals we get an almost simple strict spine $Q \subset P$ with the same vertices as $P$. Therefore $c_{\mathrm{str}}^{\mathrm{alm}}(M) \leqslant c^{\mathrm{alm}}(M)$.

Finally, note that we have $c_{\text {str }}(M)>c(M)$ in some cases. That is, a manifold $M$ may not have a simple strict spine with $c(M)$ vertices: for instance, $S^{2}$ and $D^{2}$ do not have a simple strict spine at all, and hence $c_{\text {str }}\left(S^{2}\right)=+\infty$; we also have $c_{\text {str }}\left(S^{3}\right)=1$ (the abalone is a strict spine with one vertex) and $c_{\text {str }}\left(S^{n}\right)=0$ for every $n \geqslant 4$ (a generalized Bing's house without vertices in dimension $n \geqslant 4$ is constructed in [27]). A reasonable complexity should be zero on spheres, so we do not investigate $c_{\text {str }}$ here.

\section{Drilling spheres, handles, surgery and connected sums}

We can finally employ the techniques introduced in the previous sections to study how complexity changes under the most common topological operations. 


\subsection{Drilling}

We consider first the effect of drilling along curves. Let $M$ be a compact manifold and $\gamma \subset M$ a properly embedded 1 -manifold. The drilled manifold $M_{\gamma}$ is $M_{\gamma}=$ $\overline{M \backslash R(\gamma)}$ for some regular neighborhood $R(\gamma)$.

Theorem 8.1 Let $M^{n}$ be a compact manifold of dimension $n \geqslant 4$, and $\gamma \subset M^{n}$ a properly embedded 1 -manifold. We have $c\left(M_{\gamma}^{n}\right) \leqslant c\left(M^{n}\right)$.

Proof Let $P$ be a minimal simple spine of $M$. If $\gamma$ lies in $\operatorname{int}(M)$, we can isotope it inside $P$ and disjoint from the 1 -skeleton (because $\operatorname{dim} P \geqslant 3$ ). By drilling $P$ along $\gamma$ we thus get a spine $Q$ for $M_{\gamma}$. Lemma 6.1 implies that $Q$ has no more vertices than $P$, and hence $c\left(M_{\gamma}\right) \leqslant c(M)$.

If $\gamma$ intersects $\partial M$, we can isotope it so that $\gamma=\gamma^{\prime} \cup \lambda$ where $\gamma^{\prime}$ lies in $P$ as before and $\lambda$ consists of arcs connecting $P$ to $\partial M$, and each arc is a fiber of a collar of $P$; see Figure 18.

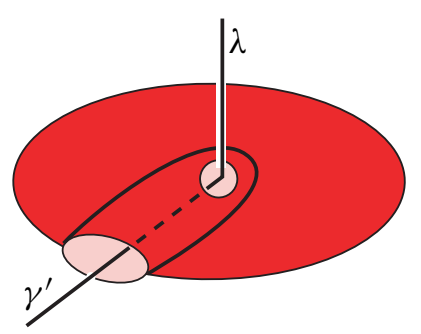

Figure 18: Put $\gamma$ as $\gamma^{\prime} \cup \lambda$ with $\gamma^{\prime} \subset P$ and $\lambda$ vertical. Then drill along $\gamma^{\prime}$ and make a hole around $\lambda$.

A spine $P^{\prime}$ of $\overline{M \backslash R\left(\gamma^{\prime}\right)}$ is constructed from $P$ by drilling along $\gamma^{\prime}$. The polyhedron $P^{\prime}$ intersects $\lambda$ transversely in some points. An almost simple spine of $M_{\gamma}$ is $P^{\prime}$ with some small open balls removed around these points. By Theorem 7.5 we get $c\left(M_{\gamma}^{n}\right) \leqslant c\left(M^{n}\right)$.

The condition $n \geqslant 4$ is necessary: in dimension 3 there is no general estimate relating $c\left(M_{\gamma}\right)$ and $c(M)$. If $M$ is closed and $c(M)>0$, it is always possible to decrease the complexity by some appropriate drilling.

Theorem 8.2 Let $M^{n}$ be a closed manifold with $c\left(M^{n}\right)>0$ and $n \geqslant 2$. There is a simple closed curve $\gamma \subset M^{n}$ such that $c\left(M_{\gamma}^{n}\right)<c\left(M^{n}\right)$. 
Proof Let $P$ be a minimal spine of $M$. Since $c(M)>0$, it has at least one vertex $v$. Let $C$ be a $(n-1)$-component of $P$ incident to $v$. By removing a small open $(n-1)$-ball from $C$ and then collapsing as more as possible we get an almost simple polyhedron $Q \subset P \subset M$ with strictly less vertices than $P$, since $v$ has been "killed" during the collapse.

The component $C$ is adjacent to one or two distinct components of $M \backslash P$. Each such component is an $n$-ball. If it is adjacent to two distinct balls, these glue to form a single ball in $M \backslash Q$, and hence $Q$ is a spine of $M$ : a contradiction, since it has less vertices than $P$. Therefore $C$ is adjacent to a single ball, and $Q$ is a spine of $M_{\gamma}$ where $\gamma \subset M$ is a closed curve intersecting $P$ transversely in one point of $\sigma$. See an example in Figure 19.

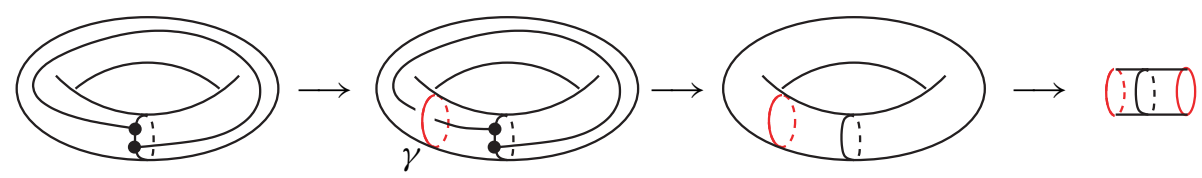

Figure 19: By making a hole on a $(n-1)$-component $C$ of $P$ we get a spine of $M \gamma$ with $\gamma$ intersecting $P$ transversely in one point. After collapsing, we kill all the vertices adjacent to $C$.

In other words, every closed manifold of positive complexity is obtained by filling a manifold of strictly smaller complexity.

Remark 8.3 In contrast with the previous result, Theorem 8.2 also holds in dimension 3. For instance, a lens space (which may have arbitrarily high complexity) is obtained by filling a solid torus, which has complexity zero. The Matveev-FomenkoWeeks smallest closed hyperbolic 3-manifold has complexity 9 [29;26] and can be obtained by filling the figure-eight knot sibling, which has complexity 2 [9]. Note the hyperbolic volume satisfies the opposite inequality $\operatorname{Vol}\left(M_{\gamma}^{3}\right)>\operatorname{Vol}\left(M^{3}\right)$ for any $\gamma$.

\subsection{Handles}

Theorem 8.4 Let $N^{n}$ be obtained from $M^{n}$ by adding a handle of index $i$.

- If $i=n$ and $n \geqslant 3$, we have $c\left(N^{n}\right)=c\left(M^{n}\right)$.

- If $i=n-1$ and $n \geqslant 4$, we have $c\left(N^{n}\right) \geqslant c\left(M^{n}\right)$.

- If $i<n-1$, we have $c\left(N^{n}\right) \leqslant c\left(M^{n}\right)$. 
Proof Suppose $i=n$. A spine $P$ of $M$ is also a spine of $N$ : hence $c(N) \leqslant c(M)$. Conversely, we can easily construct a spine of $M$ from a simple spine $P$ of $N$ with the same number of vertices by drilling around a point of $P$ not contained in the 1 -skeleton (which exists since $n \geqslant 3$ ); see Lemma 6.1.

Suppose $i=n-1$. The inverse operation of attaching a $(n-1)$-handle is drilling along the cocore arc of the handle: the inequality follows from Theorem 8.1.

We are left with the case $i<n-1$. Let $P$ be a minimal simple spine of $M$, that is a spine with $c(M)$ vertices. By using a collar for $P$ (see Section 4.2), we attach the core disc $D^{i}$ of the handle directly to $P$ and get a spine $P \cup D^{i}$ of $N$. Then $c(N) \leqslant c(M)$ by Lemma 7.4 .

\subsection{Drilling along spheres}

In Section 8.1 we showed the effect of drilling along a curve. Drilling along higherdimensional spheres gives the opposite inequality. As above we set $M_{S}=\overline{M \backslash R(S)}$. In the following, the sphere $S \subset M$ is PL but not necessarily locally flat.

Corollary 8.5 Let $M$ be a manifold and $S \subset \operatorname{int}(M)$ a $k$-sphere with $k \geqslant 2$. We have $c\left(M_{S}\right) \geqslant c(M)$.

Proof The manifold $M$ is obtained from $M_{S}$ by adding a $(n-k)$-handle and a $n-$ handle: the result then follows from Theorem 8.4. To prove the first assertion, represent $R(S)$ as a block bundle [36] over $S$ (block bundles play the rôle of normal bundles in the PL category). A $n$-block over a $k$-simplex $\sigma$ of $S$ is a $(n-k)$-handle $H$. The complement $\overline{R(S) \backslash H}$ collapses onto the disc $\overline{S \backslash \sigma}$, and hence to a point: it is therefore a disc, ie a $n$-handle.

\subsection{Surgery}

Let $M^{n}$ be a manifold of dimension $n \geqslant 2$. A surgery along a simple closed curve $\gamma \subset M^{n}$ whose regular neighborhood is homeomorphic to $D^{n-1} \times S^{1}$ consists of substituting this regular neighborhood with $S^{n-2} \times D^{2}$ via some gluing map on the boundaries, both homeomorphic to $S^{n-2} \times S^{1}$.

Corollary 8.6 Let $n \geqslant 4$ and $N^{n}$ be obtained from a closed $M^{n}$ via surgery along some closed curve. We have $c\left(N^{n}\right) \leqslant c\left(M^{n}\right)$.

If $c\left(M^{n}\right)>0$, there is a closed curve $\gamma$ such that $c\left(N^{n}\right)<c\left(M^{n}\right)$ for any manifold $N^{n}$ obtained from $M^{n}$ via surgery along $\gamma$.

Proof A surgery consists of drilling along the curve, and then adding a 2-handle and a $n$-handle. Therefore the result follows from Theorems 8.1, 8.2 and 8.4. 
Corollary 8.7 Every closed manifold $M^{n}$ of dimension $n \geqslant 4$ can be transformed into a manifold with complexity zero after at most $c(M)$ surgeries along simple closed curves.

When $M^{n}$ is simply connected and has dimension $n \geqslant 4$, a surgery is just a connected sum with either $S^{2} \times S^{n-2}$ or $S^{2} \tilde{\times} S^{n-2}$. In dimension $n \neq 4$ there is no simply connected manifold $M$ of positive complexity; see Theorem 12.1. We do not know if there is one such manifold in dimension 4. If so, the following holds.

Corollary 8.8 If $M^{4}$ is closed simply connected and $c\left(M^{4}\right)>0$, then

$$
c\left(M^{4} \#\left(S^{2} \times S^{2}\right)\right)<c\left(M^{4}\right), \quad c\left(M^{4} \#\left(S^{2} \tilde{\times} S^{2}\right)\right)<c\left(M^{4}\right) .
$$

\subsection{Connected sums}

Complexity is subadditive with respect to connected sums.

Theorem 8.9 Let $M^{n} \# N^{n}$ be obtained from $M^{n}$ and $N^{n}$ via (boundary) connected sum. If $n \geqslant 3$ we have

$$
c\left(M^{n} \# N^{n}\right) \leqslant c\left(M^{n}\right)+c\left(N^{n}\right) .
$$

Proof Making a connected sum corresponds to removing two $n$-handles from the (disconnected) manifold $M_{1} \sqcup M_{2}$, adding one 1-handle and one $n$-handle. None of these operations can increase the complexity when $n \geqslant 3$. Similarly, a $\partial$-connected sum is the addition of one 1-handle.

Complexity is actually additive on connected sums in dimension $n=3$ [29]. Actually, we do not know any example of nonadditivity in higher dimension. If there were a closed simply connected 4-manifold with $c(M)>0$, then Corollary 8.8 would yield a nonadditive connected sum.

\section{Coverings and products}

We study how complexity changes under finite coverings and products.

\subsection{Coverings}

Theorem 9.1 Let $p: \widetilde{M} \rightarrow M$ be a $d$-sheeted covering. We have $c(\widetilde{M}) \leqslant d \cdot c(M)$.

Proof Let $P$ be a minimal simple spine of $M$. Then $p^{-1}(P)$ is a simple spine of $\widetilde{M}$ with $d \cdot c(M)$ vertices. 
In contrast with Gromov norm, we often get a strict inequality $c(\widetilde{M})<d c(M)$. For instance, lens spaces may have arbitrarily high complexity while the complexity of their universal covering $S^{3}$ is zero. The following consequence is worth mentioning.

Corollary 9.2 If $M$ has complexity zero, every finite covering of $M$ has complexity zero.

\subsection{Products}

We do not know whether there is some general inequality which relates the complexity $c(M \times N)$ of a product with the complexities $c(M)$ and $c(N)$ of the factors. However, we have the following.

Theorem 9.3 Let $M^{m}, N^{n}$ be compact manifolds with $m, n \geqslant 1$. If $M$ has boundary then

$$
c\left(M^{m} \times N^{n}\right)=0 .
$$

Proof Let $P$ be any strict simple spine of $M$ (no balls in the complement, ie $M$ collapses onto $P$; see Section 7.2). Then $M \times N$ collapses onto $P \times N$. Moreover, $P \times N$ is simple without vertices: if $x \in P$ is of type $k$, a point $(x, y) \in P \times N$ is of type $k+n>0$. Therefore $c(M \times N)=0$.

Corollary 9.4 We have $c\left(S^{m} \times N\right)=0$ for every $m \geqslant 2$ and every manifold $N$.

Proof If $N$ has boundary we are done by Theorem 9.3. Otherwise, let $N^{\prime}$ be $N$ with a ball removed. We have $c\left(S^{m} \times N^{\prime}\right)=0$, and $S^{m} \times N^{\prime}$ is obtained from $S^{m} \times N$ by drilling along a $m$-sphere: Corollary 8.5 gives $c\left(S^{m} \times N\right)=0$.

\section{Normal hypersurfaces}

When $n=3$, Matveev proved [29] that complexity is nonincreasing when cutting a 3 -manifold along an incompressible surface. This was done by putting the surface in normal position with respect to the handle decomposition induced by a minimal spine, and by showing that a simple spine can be "cut" along a normal surface.

To extend this result, we define here normal discs in simplices $\Delta$ of any dimension. These can be used to define normal hypersurfaces with respect to triangulations or simple spines in any dimension: we do this in the simple case where every simplex contains at most one normal disc. In the dual setting of simple spines, this means that the normal surface is actually a subpolyhedron of the spine. 


\subsection{Normal discs}

We extend the usual definition of normal discs in a tetrahedron, used in 3-dimensional topology, to all dimensions. Let $\Delta=\Delta^{n+1}$ be the $(n+1)$-simplex.

Definition 10.1 A normal disc in $\Delta$ is a subpolyhedron dual to some partition $\mathcal{P}=$ $\left\{V_{1}, V_{2}\right\}$ of the vertices of $\Delta$ into two nonempty subsets; see Definition 5.1.

By Proposition 5.2 a normal disc is homeomorphic to $\Pi_{n}^{n} \cong D^{n}$ and is hence indeed a disc. It is a subcomplex of the subdivided $\Delta^{\prime}$. The type of a normal disc is the unordered pair $\left(\# V_{1}, \# V_{2}\right)$.

\section{Remark 10.2 There are}

$$
\left(\begin{array}{c}
n+2 \\
\# V_{1}
\end{array}\right)
$$

distinct normal discs of type $\left(\# V_{1}, \# V_{2}\right)$, except when $\# V_{1}=\# V_{2}$ : in this case there are half of them.

Remark 10.3 The 3-simplex contains the usual 7 normal discs: 4 normal triangles of type $(3,1)$ and 3 squares of type $(2,2)$; see Figure 20 . The $4-$ simplex contains
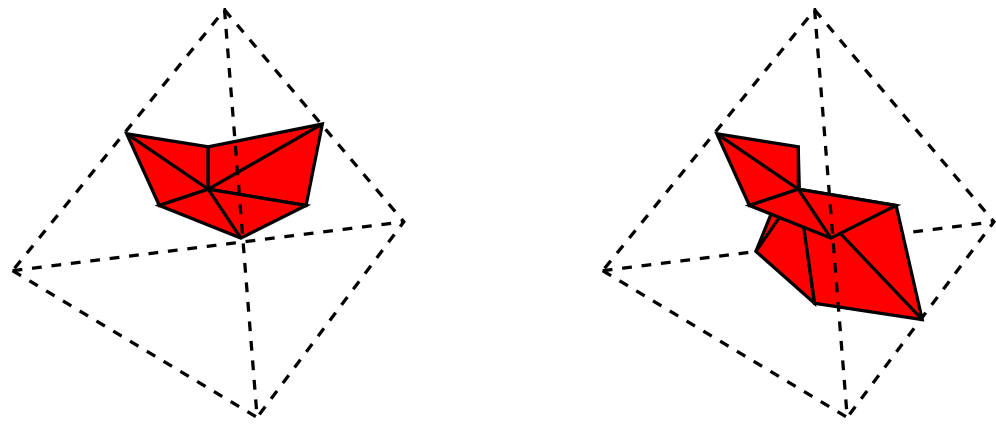

Figure 20: In dimension 3 the normal discs are the usual normal triangles and squares. Each is a subcomplex of $\Pi^{2}$, dually embedded in the tetrahedron $\Delta$.

15 normal discs: 5 normal tetrahedra of type $(4,1)$ and 10 normal dipyramids (two tetrahedra attached along one face) of type $(3,2)$. 


\subsection{Homology}

The homology group $H_{n}\left(P^{n} ; \mathbb{Z} / 2 \mathbb{Z}\right)$ of a simple polyhedron $P^{n}$ can be naturally interpreted as the set of all closed $n$-submanifolds of $P$. We prove this fact first locally and then globally.

Represent $\Pi^{n} \subset \Delta$ dually to $\Delta=\Delta^{n+1}$. A normal disc $D$ in $\Delta$ is contained in $\Pi^{n}$. Its boundary $\partial D=D \cap \partial \Pi^{n}$ is a $(n-1)$-sphere. Consider the maps

$$
\left\{\begin{array}{c}
\text { normal } \\
\text { discs in } \Delta
\end{array}\right\} \stackrel{\partial}{\longrightarrow}\left\{\begin{array}{c}
\text { closed submanifolds in } \\
\partial \Pi^{n} \text { of dimension } n-1
\end{array}\right\} \stackrel{[\cdot]}{\longrightarrow} H_{n-1}\left(\partial \Pi^{n} ; \mathbb{Z} / 2 \mathbb{Z}\right) .
$$

Of course $[\cdot]$ sends a manifold $\Sigma$ to its class $[\Sigma]$.

Proposition 10.4 If $n \geqslant 2$, both maps are bijections.

Proof Since $\operatorname{dim} \partial \Pi^{n}=n-1$, a cycle $\alpha \in H_{n-1}\left(\partial \Pi^{n} ; \mathbb{Z} / 2 \mathbb{Z}\right)$ is represented by a unique subpolyhedron $Y \subset \partial \Pi^{n} \subset S^{n}$. We must prove that $Y=\partial D$ for a normal disc $D$, induced by some partition of $V$ into two sets.

The polyhedron $Y$ is the closure of the union of some $(n-1)$-components of $\partial \Pi^{n}$. Since $Y$ is a cycle, each $(n-2)$-component of $\partial \Pi^{n}$ is adjacent to either 0 or 2 such $(n-1)$-components. As in the proof of Proposition 5.8, we conclude that $Y$ is dual to some partition $\mathcal{P}$ of the vertices in $\Delta$. If $\mathcal{P}$ contains more than 2 sets, there is a $(n-2)$-component adjacent to three $(n-1)$-components, which is forbidden. Therefore $\mathcal{P}$ consists of two sets and $Y$ is a normal disc.

Corollary 10.5 Let $P^{n}$ be a simple polyhedron. Closed $n$-submanifolds of $P$ are in natural bijection with $H_{n}\left(P^{n}, \mathbb{Z} / 2 \mathbb{Z}\right)$.

Proof Take a triangulation of $P$. Every cycle in $H_{n}(P, \mathbb{Z} / 2 \mathbb{Z})$ is represented by a subcomplex $S \subset P$. It intersects the link of every point $x$ in $P$ into a cycle: by Proposition 10.4 this is a sphere and the star of $S$ in $x$ is a normal disc. Therefore $S$ is submanifold. Different submanifolds yield different subcomplexes and hence different cycles.

\subsection{Cutting along normal surfaces}

Let $S \subset M$ be a closed submanifold in $M$ of codimension 1. As above, we set $M_{S}=\overline{M \backslash R(S)}$. A key property of 3-dimensional complexity, proved by Matveev in [29], is that $c\left(M_{S}\right) \leqslant c(M)$ whenever $S$ is an incompressible surface in a $3-$ manifold $M$. We prove here a kind of generalization of this fact. 
The powerful notion of incompressible surface unfortunately does not extend easily to higher dimensions. On the other hand, in dimension 3, every homology class in $\mathrm{H}_{2}(M, \mathbb{Z})$ is represented by an incompressible surface. We propose the following generalization of Matveev's result.

Theorem 10.6 Let $M^{n}$ be a compact manifold. Every element in $H_{n-1}\left(M^{n} ; \mathbb{Z} / 2 \mathbb{Z}\right)$ is represented by a submanifold $S$ such that $c\left(M_{S}^{n}\right) \leqslant c\left(M^{n}\right)$.

Proof Let $Q$ be a minimal simple spine of $M$. The map

$$
i_{*}: H_{n-1}(Q ; \mathbb{Z} / 2 \mathbb{Z}) \rightarrow H_{n-1}(M ; \mathbb{Z} / 2 \mathbb{Z})
$$

is surjective, because $M \backslash Q$ consists of balls and a collar of $\partial M$. Corollary 10.5 then implies that every cycle $\alpha$ is represented by a closed submanifold $S \subset Q$.

Let $P$ be obtained from $Q$ by drilling along $S$; see Section 6 . The polyhedron $P$ is a simple spine of $M_{S}$ : however, $S$ intersects the 1 -skeleton of $Q$, so we cannot use Lemma 6.1 to conclude that $c\left(M_{S}\right) \leqslant c(M)$.
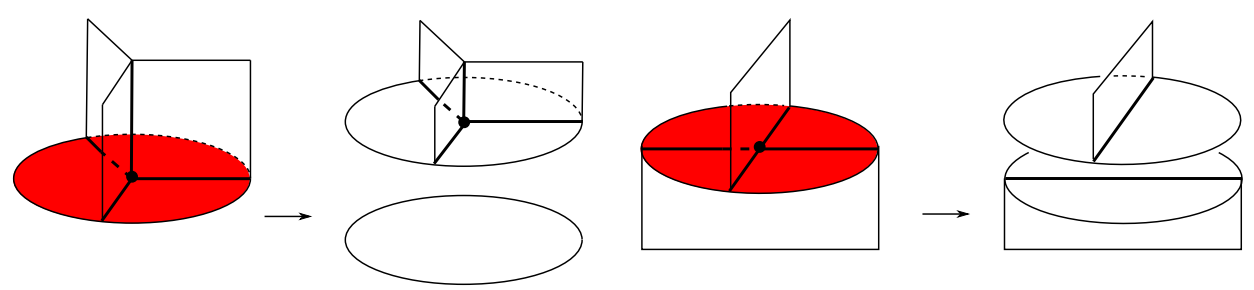

Figure 21: When the normal disc $D$ is of type $(n, 1)$, there is an adjacent 1 -component not lying in $D$. Therefore the drilling deletes a vertex and produces a new one (left). If $D$ is of some other type, it contains all the adjacent 1-components: therefore a vertex is deleted and none is created (right). Here, $n=3$.

The manifold $S$ is the union of the closure of some $(n-1)$-components of $Q$. Let $R$ be a small regular neighborhood of $S$. The proof of Lemma 6.1 shows that the new vertices of $P$ lie in the transverse intersection of the hypersurface $\partial R$ with the 1 -skeleton of $Q$. There is precisely one such intersection for every pair $(v, e)$ such that $v$ is a vertex of $Q$ contained in $S$ and $e$ is an oriented edge (ie 1-component) exiting from $v$ and not contained in $S$. Here $\partial R$ intersects $e$ transversely near $v$.

Let $(v, e)$ be one such pair. The star of $v$ in $(M, Q)$ is homeomorphic to $\left(\Delta^{n}, \Pi^{n-1}\right)$ and it intersects $S$ into a normal disc $D \subset \Pi^{n-1}$, determined by some partition $\mathcal{P}=\left\{V_{1}, V_{2}\right\}$ of the vertices of $\Delta^{n}$. The edge $e$ is dual to a facet of $\Delta^{n}$. Since $e \not \subset D$, all the $n$ vertices lying in this facet belong to the same set of the partition. Therefore 
the normal disc is of type $(n, 1)$ as in Figure 21-left. As in Figure 21-left, one new vertex is created but $v$ is destroyed: the total number of vertices in the spine does not increase.

\section{Nerve}

We study here the nerve $\mathcal{N}$ of a pair $(M, P)$ consisting of a closed manifold $M$ and a simple spine $P \subset \operatorname{int}(M)$; see Section 2.5. We prove that the nerve map $\varphi: M \rightarrow|\mathcal{N}|$ induces an isomorphism on fundamental groups when $\pi_{1}(M)$ has no torsion. Therefore $\mathcal{N}$ carries many topological information on $M$.

We have $\operatorname{dim}|\mathcal{N}| \leqslant \operatorname{dim} M=n$, and $\operatorname{dim}|\mathcal{N}|<n$ precisely if $P$ has no vertices. Actually, the $n$-dimensional part of $|\mathcal{N}|$ has a kind of singular triangulation (see Remark 5.6), with one singular $n$-simplex "dual" to each vertex of $P$.

The facts listed in Section 11.1 will be used to prove most of the results stated in Sections 12, 13 and 14. The information collected in Sections 11.2 and 11.3 are only needed to prove Theorem 13.2.

\subsection{Basic properties}

We will need the following lemma.

Lemma 11.1 Let $P$ be a spine of a compact $M$. Let $C$ be a component of $(M, P)$. The image of $i_{*}: \pi_{1}(C) \rightarrow \pi_{1}(M)$ is either finite or has a finite-index subgroup contained in the image of $i_{*}: \pi_{1}(N) \rightarrow \pi_{1}(M)$ for some boundary component $N \subset \partial M$.

Proof Consider the cut map $f: M_{P} \rightarrow M$; see Section 4.2. Let $\widetilde{C}$ be a connected component of $f^{-1}(C)$. The restriction of $f$ to $\widetilde{C}$ is a finite covering $f: \widetilde{C} \rightarrow C$. We have the following commutative diagram (with appropriate basepoints):

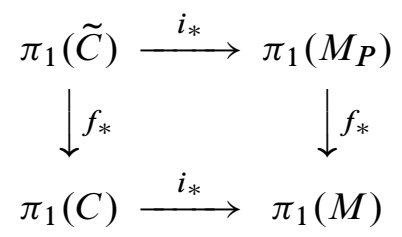

By Proposition 4.4, every component of $M_{P}$ is either a disc or a product which maps to a collar of a component $N$ of $\partial M$. Therefore $\left(f_{*} \circ i_{*}\right)\left(\pi_{1}(\widetilde{C})\right)$ is either trivial or contained in $i_{*}\left(\pi_{1}(N)\right)$. Since $f: \widetilde{C} \rightarrow C$ is a covering, the subgroup $f_{*}\left(\pi_{1}(\widetilde{C})\right)$ has finite index in $\pi_{1}(C)$ : therefore $i_{*}\left(\pi_{1}(C)\right)$ is a finite extension of $\left(i_{*} \circ f_{*}\right)\left(\pi_{1}(C)\right)=\left(f_{*} \circ i_{*}\right)\left(\pi_{1}(\widetilde{C})\right)$. 
Following Gromov [17], a set $X \subset M^{n}$ in a manifold $M^{n}$ is amenable if for every path-connected component $X^{\prime}$ of $X$ the image of the map $i_{*}: \pi_{1}\left(X^{\prime}\right) \rightarrow \pi_{1}(M)$ is an amenable group. Recall that a group $G$ is amenable if its action on the space of bounded functions $G \rightarrow \mathbb{R}$ has a left invariant mean; see Gromov [17]. Simple examples of amenable groups are finite and abelian groups. Subgroups and finite extensions of amenable groups are amenable.

Proposition 11.2 Let $P$ be a spine of a compact $M$. Let $\varphi: M \rightarrow|\mathcal{N}|$ be a nerve map of $(M, P)$.

(1) If $M$ is closed or with amenable boundary, the fiber $\varphi^{-1}(x)$ of every point $x \in|\mathcal{N}|$ is amenable.

(2) If $M$ is closed and $\pi_{1}(M)$ is torsion-free, the map $\varphi_{*}: \pi_{1}(M) \rightarrow \pi_{1}(|\mathcal{N}|)$ is an isomorphism.

Proof The fiber $\varphi^{-1}(x)$ of a point is contained in some component of $(M, P)$, since each fiber of the prenerve map does; see Section 2.5. Since subgroups and finite extensions of amenable groups are amenable, point (1) follows from Lemma 11.1.

We turn to (2). The map $\varphi: \mathcal{M} \rightarrow|\mathcal{N}|$ is surjective and the fiber $\varphi^{-1}(x)$ over a point of $|\mathcal{N}|$ is path-connected: these two facts imply that $\varphi_{*}$ is surjective. On the other hand, Lemma 11.1 implies that $i_{*}\left(\varphi^{-1}(x)\right)<\pi_{1}(M)$ is a finite group. If $\pi_{1}(M)$ has no torsion, this finite group is trivial: this easily implies that $\varphi_{*}$ is injective.

Remark 11.3 We note that Proposition 11.2 (2) does not hold in general for the prenerve map $\varphi_{0}: M \rightarrow\left|\mathcal{N}_{0}\right|:$ we really need Stein factorization here. See Exercise 2.3 and Figure 22.
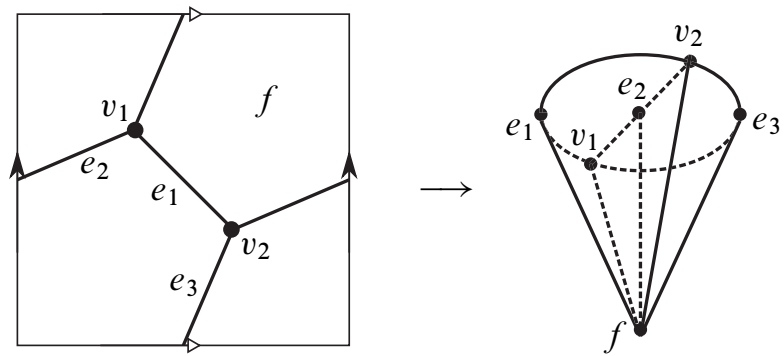

Figure 22: The prenerve of a spine $P$ of the torus $T$ with two vertices is homeomorphic to the cone over $P$, and is hence simply connected: the vertex of the cone is the 2-component $f=T \backslash P$, which is incident to all the other components. 
Remark 11.4 Some hypothesis on $\pi_{1}(M)$ is indeed necessary in Proposition 11.2 (2). For instance, a hyperplane $H \subset \mathbb{R} \mathbb{P}^{n}$ is a spine of $\mathbb{R} \mathbb{P}^{n}$, but the nerve of $\left(\mathbb{R P} \mathbb{P}^{n}, H\right)$ is a segment: hence $\varphi_{*}: \mathbb{Z} / 2 \mathbb{Z} \rightarrow\{e\}$ is not an isomorphism.

Remark 11.5 Let $P$ be a spine of a closed manifold $M$ dual to some triangulation $T$ as in Section 5.2 (with the discrete partition $v \sim v^{\prime} \Leftrightarrow v=v^{\prime}$ ). The prenerve map $\varphi: T^{\prime} \rightarrow \mathcal{N}_{0}$ is an isomorphism. Therefore $\mathcal{N} \cong \mathcal{N}_{0}^{\prime} \cong T^{\prime \prime}$ and the nerve map $\varphi: M \rightarrow|\mathcal{N}|$ is a homeomorphism.

Concerning the dimension of the nerve, we have the following.

Proposition 11.6 Let $\mathcal{N}$ be the nerve of a pair $(M, P)$. We have $\operatorname{dim} \mathcal{N} \leqslant \operatorname{dim} M$. We have $\operatorname{dim} \mathcal{N}=\operatorname{dim} M$ if and only if $P$ has vertices.

Proof Set $n=\operatorname{dim} M$. Let $\mathcal{N}_{0}$ be the prenerve of $(M, P)$. We have $\operatorname{dim} \mathcal{N}=\operatorname{dim} \mathcal{N}_{0}$. A $k$-simplex in $\mathcal{N}_{0}$ is determined by some components $C_{0}<\ldots<C_{k}$. In particular, we have $0 \leqslant \operatorname{dim} C_{0}<\cdots<\operatorname{dim} C_{k} \leqslant n$. This implies that $\operatorname{dim} \mathcal{N}_{0} \leqslant n$. If $P$ has no vertices, then $\operatorname{dim} C_{0} \geqslant 1$ and hence $\operatorname{dim} \mathcal{N}_{0}<n$. If $P$ has a vertex $v$, there is a chain $\{v\}=C_{0}<\cdots<C_{n}$ and hence $\operatorname{dim} \mathcal{N}=n$.

\subsection{Fundamental class}

Let $P \subset \operatorname{int}(M)$ be any simple polyhedron in a compact manifold (possibly with boundary). Let $\varphi: M \rightarrow|\mathcal{N}|$ be a nerve map of $(M, P)$. We show that $\mathcal{N}$ looks like a pseudomanifold. In particular, we can define a notion of fundamental class.

Proposition 11.7 Every $(n-1)$-simplex in $\mathcal{N}$ is adjacent to either zero or two $n-$ simplices.

Proof Let $\sigma$ be a $(n-1)$-simplex in $\mathcal{N}$. The counterimage $\varphi^{-1}\left(\sigma_{*}\right)$ of its barycenter $\sigma_{*}$ is either a point or a connected $1-$ manifold in $\operatorname{int}(M)$. If it is a point or a segment then $\sigma$ is adjacent to two $n$-simplices. If it is a circle then $\sigma$ is not adjacent to any $n$-simplex.

The nerve $\mathcal{N}$ looks like a pseudomanifold. Note however that the adjacencies of the $n$-simplices along the $(n-1)$-simplices do not necessarily form a connected graph: for instance, $|\mathcal{N}|$ might consist of a bouquet of two $n$-manifolds.

A fundamental class for $\mathcal{N}$ is an element of $H_{n}(|\mathcal{N}|, \mathbb{Z})$ which may be written (in simplicial homology) as a sum of all $n$-simplices of $\mathcal{N}$, each with an appropriate sign \pm 1 . We indicate such a class as $[\mathcal{N}]$. A fundamental class induces an orientation on each $n$-simplex of $\mathcal{N}$. 
Proposition 11.8 If $M$ is oriented then $[\mathcal{N}]=\varphi_{*}([M, \partial M])$ is a fundamental class.

Proof Let $T$ be a sufficiently subdivided triangulation of $(M, P)$ defining the nerve map $\varphi: T^{\prime} \rightarrow \mathcal{N}$. Since $\varphi$ is surjective with connected fibers, every $n$-simplex $\sigma$ in $\mathcal{N}$ is the image of precisely one $n$-simplex $\eta$ of $T^{\prime}$. The class $[M, \partial M]$ is a fundamental class, ie it is represented by a sum of all the simplices of $M$ with appropriate signs, and hence (using simplicial homology) also $\varphi_{*}([M, \partial M])$ is a sum of all the simplices of $\mathcal{N}$ with appropriate signs.

\subsection{Singular simplices}

Let $P \subset \operatorname{int}(M)$ be any simple polyhedron in a manifold and $\varphi: M \rightarrow|\mathcal{N}|$ a nerve map. We have just seen that $\mathcal{N}$ looks like a pseudomanifold. We now prove that the $n$-dimensional part of $\mathcal{N}$ is the twice barycentric subdivision of a singular triangulation (see Remark 5.6), with one singular simplex corresponding "dually" to each vertex of $P$.

Let $v$ be a vertex of $P$. A local component at $v$ is a component of a fixed open star of $v$ in $(M, P)$. The open star is homeomorphic to $\operatorname{int}\left(\Delta, \Pi^{n-1}\right)$. In the dual representation, the polyhedron $\Pi^{n-1}$ is a subcomplex of $\Delta^{\prime}$ and the components of $\operatorname{int}\left(\Delta, \Pi^{n-1}\right)$ are naturally identified with the vertices of $\Delta^{\prime}$.

Every local component is contained in a unique component of $(M, P)$, so we get a simplicial map $\beta_{v}: \Delta^{\prime} \rightarrow \mathcal{N}_{0}$. The map $\beta_{v}$ is topologically a singular simplex in $\left|\mathcal{N}_{0}\right|$. It sends the barycenter of $\Delta$ to the component $\{v\}$. The image of $\beta_{v}$ is precisely the star of $\{v\}$ in $\mathcal{N}_{0}$.

We want a singular simplex in $|\mathcal{N}|$. We therefore try to lift $\beta_{v}$ to $\mathcal{N}$ along the projection $g: \mathcal{N} \rightarrow \mathcal{N}_{0}^{\prime}$. The following result says that there is a natural way do to this.

Proposition 11.9 There is a natural simplicial map $\alpha_{v}: \Delta^{\prime \prime} \rightarrow \mathcal{N}$ such that $g \circ \alpha_{v}=\beta_{v}^{\prime}$.

Proof We can formalize this as follows. The restriction of $\varphi_{0}$ to the (closed) star of $v$ in $T$ splits naturally into two simplicial maps

$$
\operatorname{st}(v, T) \stackrel{\varphi_{v}}{\longrightarrow} \Delta^{\prime} \stackrel{\beta_{v}}{\longrightarrow} \mathcal{N}_{0} .
$$

The map $\varphi_{v}$ sends every vertex to the local component to which it belongs. The map $\varphi_{v}$ has connected fibers. Therefore there is a unique simplicial map $\alpha_{v}: \Delta^{\prime \prime} \rightarrow \mathcal{N}$ 
which makes the diagram

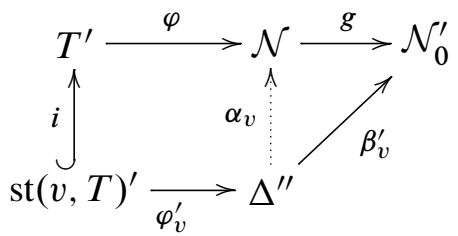

commute, completing the proof.

The map $\alpha_{v}$ is actually well-defined only up to a permutation of the vertices of $\Delta$, induced from the chosen identification of the open star of $v$ with $\operatorname{int}\left(\Delta, \Pi^{n-1}\right)$.

The following propositions show that the singular simplices $\alpha_{v}$ at the varying of $v$ among the vertices of $P$ form a singular triangulation of the $n$-dimensional part of $|\mathcal{N}|$ whose double barycentric subdivision yields the original triangulation of $\mathcal{N}$.

Proposition 11.10 If $v \neq v^{\prime}$ then $\alpha_{v}(\operatorname{int}(\Delta)) \cap \alpha_{v^{\prime}}(\operatorname{int}(\Delta))=\varnothing$.

Proof It suffices to prove the assertion for $\beta_{v}$ and $\beta_{v^{\prime}}$. The image of int $(\Delta)$ along $\beta_{v}$ is the open star of $\{v\}$ in $\mathcal{N}_{0}$. The open stars of $\{v\}$ and $\left\{v^{\prime}\right\}$ are disjoint (because $v$ and $v^{\prime}$ are not connected by an edge).

Proposition 11.11 The map $\alpha_{v}$ is injective in $\operatorname{int}(\Delta)$ for every $v$.

Proof We have a commutative diagram of topological maps:

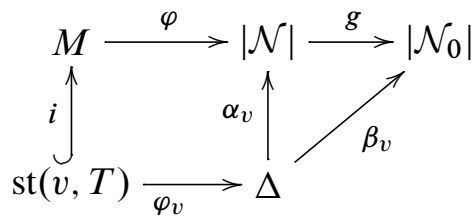

Take $x \in \operatorname{int}(\Delta)$. The image $\beta_{v}(x)$ lies in the interior of the star of $\{v\}$ in $\mathcal{N}_{0}$. Then $\varphi_{0}^{-1}\left(\beta_{v}(x)\right)$ lies in the interior of the star of $v$ in $T$. Therefore $\varphi^{-1}\left(\alpha_{v}(x)\right)$ is also contained in $\operatorname{st}(v, T)$, and is connected since $\varphi$ has connected fibers.

Therefore $\varphi_{v}\left(\varphi^{-1}\left(\alpha_{v}(x)\right)=\alpha_{v}^{-1}\left(\alpha_{v}(x)\right)\right.$ is connected. The map $\alpha_{v}$ is finite-to-one by construction, thus this set is also finite. Therefore it consists of only one point $\{x\}$.

Proposition 11.12 Every $n$-simplex of $\mathcal{N}$ is contained in $\alpha_{v}(\Delta)$ for some $v$. 
Proof Let $\sigma$ be a $n$-simplex of $\mathcal{N}$. Since the nerve map $\varphi: T^{\prime} \rightarrow \mathcal{N}$ has connected fibers, the preimage of $\sigma$ consists of a single $n$-simplex $\varphi^{-1}(\sigma)$. We show that $\varphi^{-1}(\sigma)$ lies in the star $\operatorname{st}(v, T)$ of some vertex $v$ : this implies that $\sigma$ is contained in $\alpha_{v}(\Delta)$.

Let $\bar{\sigma}$ be the $n$-simplex of $T$ which contains $\varphi^{-1}(\sigma)$. Consider the maps

$$
T^{\prime} \stackrel{\varphi}{\longrightarrow} \mathcal{N} \stackrel{g}{\longrightarrow} \mathcal{N}_{0}^{\prime} .
$$

The image $\varphi_{0}(\bar{\sigma})=g(\varphi(\bar{\sigma}))$ is a $n$-simplex because $g$ preserves the dimension of simplices. That is, the vertices of $\bar{\sigma}$ lie in distinct components $C_{0}<\cdots<C_{n}$ of $(M, P)$. This implies that $\operatorname{dim} C_{i}=i$, and thus $C_{0}=\{v\}$ is a vertex. Therefore $\bar{\sigma}$ lies in $\operatorname{st}(v, T)$.

Finally, we show that the singular simplices $\alpha_{v}$ glue in pairs along their facets. An edge of $P$ is a 1 -component not homeomorphic to a circle. An edge $e$ connects two (possibly coinciding) vertices $v_{1}$ and $v_{2}$; see Figure 23. Identify the stars of $v_{1}$ and $v_{2}$ with $\left(\Delta, \Pi^{n-1}\right)$. The edge $e$ intersects each $\Delta$ in the barycenter of a facet $f_{i}$ of $\Delta$. The edge $e$ induces a simplicial isomorphism $\psi: f_{1} \rightarrow f_{2}$ defined by taking track along $e$ of the incident components.

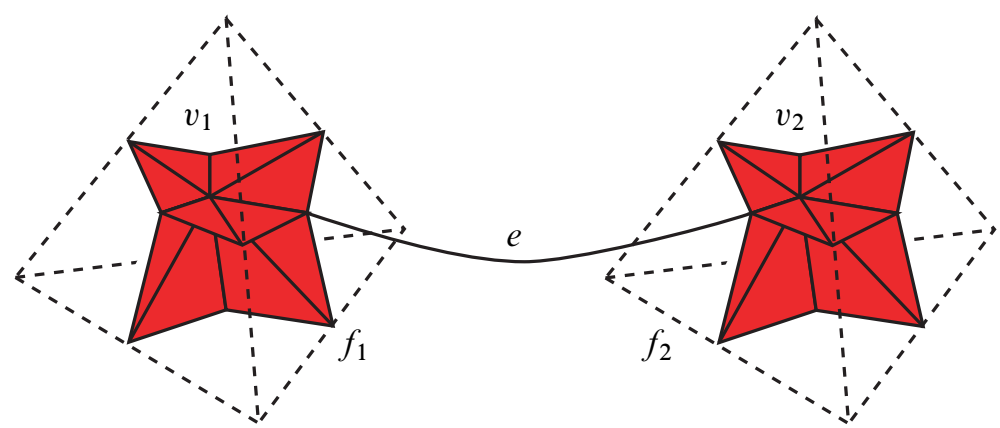

Figure 23: An edge $e$ connecting two vertices $v_{1}$ and $v_{2}$. It intersects two facets $f_{1}$ and $f_{2}$ of the dual simplices.

Proposition 11.13 We have $\left.\alpha_{v_{1}}\right|_{f_{1}}=\alpha_{v_{2}} \circ \psi$.

Proof Take

$$
R=\overline{R(e, T) \backslash\left(R\left(v_{1}, T\right) \cup R\left(v_{2}, T\right)\right)} .
$$

We can identify $R$ with a cylinder $\Delta^{n-1} \times[-1,1]$, which intersects $P$ as $\Pi^{n-2} \times[-1,1]$, such that $\varphi_{0}(x, t)$ does not depend on $t \in[-1,1]$ for every $x \in \Delta^{n-1}$. Therefore $\left.\beta_{v_{1}}\right|_{f_{1}}=\beta_{v_{2}} \circ \psi$ and the lifts $\left.\alpha_{v_{1}}\right|_{f_{1}}$ and $\alpha_{v_{2}} \circ \psi$ also coincide. 
Suppose $M$ is oriented. By Proposition 11.8 the orientation on $M$ induces a fundamental class $[\mathcal{N}]$ in $\mathcal{N}$ and hence a coherent orientation on its $n$-simplices. A singular simplex $\alpha_{v}$ can thus be orientation-preserving or reversing, and we set $\sigma_{v}=0$ or 1 correspondingly.

We would like to say that $\sum(-1)^{\sigma_{v}} \alpha_{v}$ is a cycle representing $[\mathcal{N}]$. However, this argument is wrong: a singular triangulation, without any additional data, does not yield a cycle, because boundary maps do not necessarily cancel algebraically (for that reason, the slightly richer notion of semisimplicial complex [12] or $\Delta$-complex [19, page 103] is usually employed in the literature). We solve this problem by averaging each $\alpha_{v}$ over its $(n+1)$ ! different representations. That is, we define

$$
\tilde{\alpha}_{v}=\frac{1}{(n+1) !} \sum_{p \in S_{n+1}}(-1)^{\sigma_{v}+\operatorname{sgn}(p)} \alpha_{v} \circ \theta_{p}
$$

where $\theta_{p}: \Delta \rightarrow \Delta$ is the combinatorial isomorphism induced by the permutation $p$ of the vertices of $\Delta$ (which have a fixed ordering, ie a fixed identification with $\{1, \ldots, n+1\})$. The chain $\widetilde{\alpha}_{v}$ depends only on $v$.

Proposition 11.14 Let $M$ be oriented. We have

$$
\varphi_{*}([M, \partial M])=[\mathcal{N}]=\left[\sum_{v} \tilde{\alpha}_{v}\right] .
$$

Proof First, note that $\sum_{v} \widetilde{\alpha}_{v}$ is indeed a cycle: the terms in the boundary cancel in pairs thanks to Proposition 11.13. The twice subdivision of $\sum_{v} \tilde{\alpha}_{v}$ yields the fundamental class $[\mathcal{N}]$, each simplex being counted $(n+1)$ ! times with coefficient $1 /(n+1)$ !

\section{Homotopy type}

We study here the relations between the complexity and some homotopy invariants of a manifold. First, note that the complexity is not a homotopy invariant, since it distinguishes homotopically equivalent lens spaces: we have $c\left(L_{7,1}\right)=4$ and $c\left(L_{7,2}\right)=2$ [29].

\subsection{Fundamental groups}

It might be that every simply connected manifold has complexity zero. However, this question is open in dimension 4. 
Theorem 12.1 A simply connected compact manifold of dimension $n \neq 4$ has complexity zero.

Proof A simply connected manifold of dimension $n \neq 4$ has a handle decomposition without 1-handles. In dimension 3, this follows from Perelman's proof of Poincaré Conjecture. Concerning dimension $n \geqslant 5$; see Rourke and Sanderson [37, Lemma 6.15 and the subsequent remark]. By turning upside-down the handle decomposition, we get $M$ as $\partial M \times[0,1]$ or $D^{4}$ plus some handles of index $\neq n-1$. The manifold has then complexity zero by Theorem 9.3 and Theorem 8.4.

The smallest known fundamental group of a manifold with positive complexity is $\mathbb{Z} / 4 \mathbb{Z}$ : the lens space $L_{4,1}$ has $c\left(L_{4,1}\right)=1$. We do not know if there are manifolds of positive complexity with fundamental group $\mathbb{Z} / 2 \mathbb{Z}$ or $\mathbb{Z} / 3 \mathbb{Z}$.

On the other hand, every finitely presented group is the fundamental group of a closed 4-manifold of complexity zero: see Corollary 15.2.

\subsection{Essential manifolds}

Following Gromov [18] a non-simply connected closed manifold $M$ is essential if one of the following equivalent conditions holds:

(1) The image $f_{*}([M]) \in H_{n}(K(\pi, 1))$ of its fundamental class in the corresponding Eilenberg-Mac Lane space is nontrivial.

(2) There is no map $f: M \rightarrow X$ onto a lower-dimensional polyhedron $X$ which induces an isomorphism on fundamental groups.

The equivalence between these definitions was proved by Babenko [5]. A group is virtually torsion-free if it contains a finite-index torsion-free subgroup.

Theorem 12.2 Let $M$ be a closed manifold with virtually torsion-free infinite fundamental group. If $c(M)=0$ then $M$ is not essential.

Proof There is a finite covering $\widetilde{M} \rightarrow M$ such that $\pi_{1}(\widetilde{M})$ is infinite and torsion-free. We have $c(\widetilde{M})=0$ by Corollary 9.2. Let $P$ be a spine of $\widetilde{M}$ without vertices. By Proposition 11.6, the nerve $|\mathcal{N}|$ of $(\widetilde{M}, P)$ has dimension smaller than $\operatorname{dim} \widetilde{M}$. On the other hand, the nerve map $\varphi: \widetilde{M} \rightarrow|\mathcal{N}|$ yields an isomorphism on fundamental groups by Proposition 11.2. Therefore $\widetilde{M}$ is not essential. This implies that $M$ is not essential. 
A manifold is aspherical if $\pi_{i}(M)$ is trivial for all $i>1$. Equivalently, the universal cover $\widetilde{M}$ is contractible. Closed aspherical manifolds and real projective spaces are essential. The following fact is well-known.

Proposition 12.3 An aspherical manifold has torsion-free fundamental group.

Proof Suppose that $M$ is aspherical and $\pi_{1}(M)$ contains a nontrivial finite cyclic group $C_{p}$. Let $\widetilde{M} \rightarrow M$ be the covering determined by $C_{p}$. The manifold $\widetilde{M}$ is also aspherical. Therefore $H_{i}(\widetilde{M}, \mathbb{Z}) \cong H_{i}\left(C_{p}, \mathbb{Z}\right)$. However, the group $H_{i}\left(C_{p}, \mathbb{Z}\right)$ is nontrivial for infinitely many values of $i$ : a contradiction because $M$ is a manifold. $\square$

Corollary 12.4 A closed aspherical manifold $M$ has $c(M)>0$.

In particular, the $n$-torus $S^{1} \times \cdots \times S^{1}$ has positive complexity. Products of $S^{1}$ and/or closed surfaces of positive genus also have positive complexity.

Remark 12.5 Some hypothesis on $\pi_{1}(M)$ is necessary in Theorem 12.2, since $\mathbb{R P}^{n}$ is essential and $c\left(\mathbb{R P}^{n}\right)=0$ for $n \geqslant 2$.

Remark 12.6 The same arguments show that connected sums of aspherical manifolds have positive complexity.

\section{Gromov norm}

Let $M$ be an orientable manifold, possibly with boundary. The Gromov norm $\|M\|$ of $M$ is

$$
\|M\|=\|[M, \partial M]\|=\inf \left\{\left|a_{1}\right|+\cdots+\left|a_{k}\right| \mid[M, \partial M]=\sum a_{i} \sigma_{i}\right\}
$$

where the infimum is taken among all representations of the fundamental class $[M, \partial M] \in$ $H_{n}(M, \partial M ; \mathbb{R})$ as singular cycles $\sum a_{i} \sigma_{i}$. See Gromov [17].

\subsection{Complexity zero}

Theorem 13.1 Let $M$ be closed or with amenable boundary. If $c(M)=0$ then $\|M\|=0$. 
Proof Since $c(M)=0$, our $M$ has a spine $P$ without vertices. Let $\mathcal{N}$ be the nerve of $(M, P)$. We have $\operatorname{dim} \mathcal{N}<\operatorname{dim} M$ by Proposition 11.6. Proposition 11.2 implies that $M$ fibers on a low-dimensional polyhedron with amenable fibers. Therefore $M$ can be covered by at most $n$ amenable (not necessarily connected) open sets. If $M$ is closed, Gromov's Vanishing Theorem [17] implies that $\|M\|=0$.

If $M$ has boundary, every boundary component lies in one fiber and the fibration extends naturally to the double $D M$. Therefore $\|D M\|=0$. Since $\partial M$ is amenable, we have $\|D M\|=2\|M\|$ by [21], so $\|M\|=0$.

The hypothesis on $\partial M$ is indeed necessary: for instance, a 3-dimensional handlebody of genus 2 has complexity zero and positive norm (because its boundary has positive Gromov norm).

\subsection{General inequality}

Theorem 13.2 Let $M$ be closed with virtually torsion-free $\pi_{1}(M)$. Then

$$
\|M\| \leqslant c(M) .
$$

Proof Let $\widetilde{M} \rightarrow M$ be the degree- $d$ covering induced by the torsion-free subgroup. Since $\|\widetilde{M}\|=d\|M\|$ and $c(\widetilde{M}) \leqslant d c(M)$, it suffices to prove the theorem for $\widetilde{M}$, which we still call $M$ for simplicity.

Now $\pi_{1}(M)$ is torsion-free. Let $P$ be a minimal spine of $M$, ie a spine with $c(M)$ vertices. Let $\mathcal{N}$ be the nerve of $(M, P)$. A nerve map $\varphi: M \rightarrow|\mathcal{N}|$ induces an isomorphism $\varphi_{*}: \pi_{1}(M) \rightarrow \pi_{1}(|\mathcal{N}|)$ by Proposition 11.2. Gromov's Mapping Theorem [17] says that an isomorphism on fundamental groups yields an isomorphism and isometry on bounded cohomology, and hence $\left\|\varphi_{*}(\alpha)\right\|=\|\alpha\|$ for every cycle $\alpha \in H_{*}(M)$. In particular,

$$
\|M\|=\|[M]\|=\left\|\varphi_{*}([M])\right\| .
$$

Proposition 11.14 shows a cycle that represents $\varphi_{*}([M])$ whose norm equals the number $c(M)$ of vertices of $P$. Therefore $\|M\| \leqslant c(M)$.

Theorem 13.2 is actually the only tool we have to prove the following.

Corollary 13.3 There are manifolds of arbitrarily high complexity in any dimension $n$. 


\section{Riemannian geometry}

\subsection{Hyperbolic manifolds}

Theorem 13.1 implies the following.

Corollary 14.1 Let $M$ be a compact manifold whose interior admits a complete hyperbolic metric of finite volume. Then $c(M)>0$.

Proof Each boundary component $N^{n-1} \subset \partial M^{n}$ corresponds to a cusp of $M$ and is a flat $(n-1)$-manifold. By Bieberbach Theorem $\pi_{1}(N)$ has a finite-index subgroup isomorphic to $\mathbb{Z}^{n-1}$ and is hence amenable. We have $\|M\|>0$ because $M$ admits a complete hyperbolic metric [17] and therefore $c(M)>0$ by Theorem 13.1.

This result is sharp since the Gieseking 3-manifold (nonorientable with a Klein bottle cusp) has complexity 1 [9].

Remark 14.2 We do not know if Corollary 14.1 holds for hyperbolic manifolds with geodesic boundary; it does in dimensions 2 and 3 (because every compact irreducible, $\partial$-irreducible and anannular 3-manifold has positive complexity [29]).

Theorem 13.2 in turn implies the following finiteness result.

Corollary 14.3 For every $n$ and $k$ there are finitely many closed hyperbolic manifolds $M^{n}$ with $c\left(M^{n}\right)<k$.

Proof In dimension $n=3$, there are finitely many irreducible manifolds of any given complexity [29], so we are done. In dimension $n \neq 3$ there are finitely many hyperbolic manifolds (up to homeomorphism) of bounded volume [6]. The volume of a hyperbolic manifold is proportional to its Gromov norm. Since $\pi_{1}(M)$ is torsion-free, Theorem 13.2 gives $\|M\| \leqslant c(M)$ and we are done.

Note that the same assertion for Gromov norm (or equivalently hyperbolic volume) is not true for $n=3$.

Remark 14.4 We do not know if Corollary 14.3 holds for hyperbolic manifolds with cusps and/or geodesic boundary: it does in dimensions 2 and 3 (because there are finitely many compact irreducible, $\partial$-irreducible, and anannular 3-manifolds having a fixed complexity [29]). 


\subsection{Manifolds of nonpositive curvature}

A closed Riemannian manifold with nonpositive sectional curvature is aspherical because of the Cartan-Hadamard theorem. Corollary 12.4 then implies the following.

Corollary 14.5 A closed Riemannian manifold $M$ with nonpositive sectional curvature has $c(M)>0$.

Remark 14.6 In contrast with the hyperbolic case, Corollary 14.5 does not hold for every compact manifold $M$ whose interior admits a complete metric of nonpositive (or even negative) curvature and finite volume (see Corollary 14.1). For instance, a product of a closed surface and a bounded surface, both with $\chi<0$, admits such a metric and has complexity zero by Theorem 9.3.

\subsection{Geometric invariants}

Complexity is related to other interesting geometric invariants. Let $(M, g)$ be a Riemannian manifold. The volume entropy of $(M, g)$ is the limit

$$
\lambda(M, g)=\lim _{R \rightarrow \infty} \frac{\log \operatorname{Vol}((B(p, R))}{R}
$$

where $\operatorname{Vol}(B(p, R))$ is the volume of the ball of radius $R$ around a point $p$ in the universal cover $\widetilde{M}$, taken with respect to the lifted metric $\widetilde{g}$. Such a quantity does not depend on $p$ [22]. The systole $L(M, g)$ is the length of the shortest closed geodesic which is not homotopically trivial.

The volume entropy $\lambda(M)$ and systolic constant $\sigma(M)$ of $M$ are defined respectively as the infimum of the volume entropies and systoles among all metrics $g$ on $M$ of volume 1. The spherical volume $T(M)$ is defined by Besson, Courtois and Gallot [7].

Theorem 14.7 Let $M$ be a closed orientable manifold with virtually torsion-free infinite fundamental group. If $c(M)=0$ then

$$
T(M)=\lambda(M)=\sigma(M)=0 .
$$

Proof The manifold $M$ is not essential by Theorem 12.2. Babenko showed in [5] that a nonessential manifold $M$ has $\lambda(M)=\sigma(M)=0$. Moreover $\lambda(M)=0$ implies $T(M)=0[7]$.

The hypothesis on $\pi_{1}(M)$ is necessary for the vanishing of $\sigma$, since $c\left(\mathbb{R P}^{n}\right)=0$ and $\sigma\left(\mathbb{R P}^{n}\right)>0$. We do not know if it is necessary for the vanishing of $T$ and $\lambda$. It is not necessary for the vanishing of $\|\cdot\|$ because of Theorem 13.1 . 


\subsection{Thin Riemannian manifolds}

The cut locus of a Riemannian manifold is sometimes a simple spine. Alexander and Bishop $[2 ; 3]$ proved that the cut locus of a thin Riemannian manifold is a simple spine without vertices: a thin Riemannian manifold has therefore complexity zero. This happens for instance if we assign a product metric to $M \times[0,1]$ which is very small on $[0,1]$.

Following [2], a Riemannian manifold $M$ is thin if the radii of all metric balls in $M$ are small relative to $K_{M}$ and $\kappa_{\partial M}$, where $K_{M}$ is the sectional curvature of the interior and $\kappa_{\partial M}$ is the normal curvature of the boundary $\partial M$. To get a scale-free measure of the width of a manifold, Alexander and Bishop used the curvature-normalized inradius

$$
J \cdot \max \left\{\sup \sqrt{\left|K_{M}\right|}, \sup \left|\kappa_{\partial M}\right|\right\},
$$

where $J$ is the supremum over all points in $M$ of their distances to $\partial M$.

Theorem 14.8 (Alexander-Bishop [2]) There are universal constants $a_{2}<a_{3}<\cdots$ such that if a bounded Riemannian manifold $M^{n}$ has (curvature-normalized) inradius less than $a_{n}$, then $c\left(M^{n}\right)=0$.

Proof As shown in [2], there exists a sequence of universal constants $a_{2}<a_{3}<\ldots$ such that if the curvature-normalized inradius of $M$ is less than $a_{h}$ and $h \leqslant n+1$, then the cut locus of $M$ is simple and every point is of type $\geqslant n+1-h$. Therefore it has no vertices for $h<n+1$. The cut locus is a spine, hence $c(M)=0$ in that case. $\square$

\section{Four-manifolds}

We describe here some families of 4-manifolds having complexity zero. The various results proved in this paper show that the set of all 4-manifolds of complexity zero contains all products $N \times N^{\prime}$ with nonempty boundary or $N \in\left\{S^{2}, S^{3}\right\}$ and is closed under connected sums, finite coverings, addition of handles of index $\neq 3$, and surgery along simple closed curves.

We show that Fintushel and Stern's infinitely many exotic K3's and Gompf's symplectic manifolds with arbitrary $\pi_{1}$ are constructed by adding handles of index $\neq 3$ to a product: they thus have complexity zero. The double of a 2-handlebody has also complexity zero, because it is obtained by surgering along a link in a connected sum of some copies of $S^{3} \times S^{1}$. 


\subsection{Arbitrary fundamental group}

Closed 4-manifolds with complexity zero may have arbitrary (finitely presented) fundamental groups.

Theorem 15.1 The double $D M$ of a four-manifold $M$ made of only $0-, 1-$, and 2-handles has $c(D M)=0$.

Proof The manifold $M$ can be decomposed into $0-, 1-$ and $2-$ handles. The $0-$ and 1 -handles form a 1 -handlebody $H$. The cores of the 2-handles are attached along a link $L \subset \partial H$. The double $D M$ is the result of a surgery along $L$ in $D H$. Now $D H$ is the connected sum of some copies of $S^{1} \times S^{3}$ and hence $c(D H)=0$ by Corollary 9.4 and Theorem 8.9. Then $c(D M)=0$ by Corollary 8.6.

Corollary 15.2 Every finitely presented group is the fundamental group of a closed orientable 4-manifold of complexity zero.

Proof Every finitely presented group is the fundamental group of a 4-manifold made of $0-, 1-$ and 2-handles. Doubling it does not change the fundamental group.

Remark 15.3 It is not true that every double has complexity zero. For instance, if $M$ is a product of a closed surface and a bounded surface, both with $\chi \leqslant 0$, then $D M$ is a product of closed aspherical surfaces and hence $c(D M)>0$ by Corollary 12.4. Note that $c(M)=0$ by Theorem 9.3.

\subsection{Capping with elliptic surfaces}

Consider the elliptic surface $E(n)$, described as a fiber-connected sum of $n$ copies of $E(1)$ as in [16]. The elliptic surface has an elliptic fibration $E(n) \rightarrow S^{2}$, whose regular fiber is a torus. The regular neighborhood $R(T)$ of a regular fiber $T$ is homeomorphic to a product $T \times D^{2}$. Define

$$
E^{\prime}(n)=E(n) \backslash \operatorname{int}(R(T)) .
$$

This is a simply connected manifold with a 3-torus as its boundary. It has a decomposition with only $0-$ and 2-handles [16, Figures 8.9 and 8.10]. This manifold is sometimes used to cap some boundary component of a 4-manifold; see Figure 24.

Lemma 15.4 Let $M^{3}$ be a compact 3-manifold whose boundary consists of some tori. Let $N^{4}$ be obtained by capping some boundary components of $M^{3} \times S^{1}$ via some copies of $E^{\prime}(n)$, along some maps. Then $c(N)=0$. 


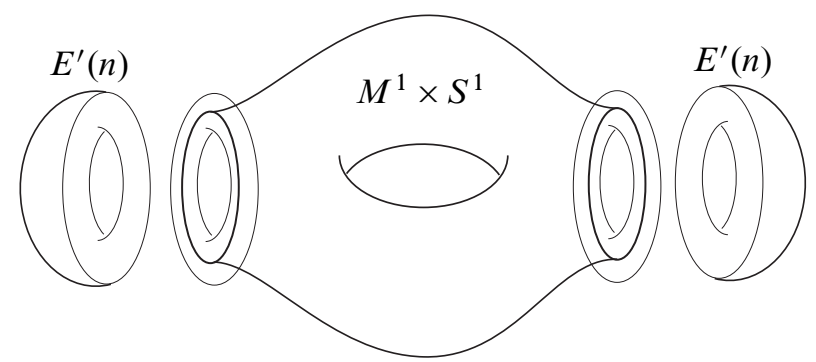

Figure 24: A manifold constructed by taking a product $M^{3} \times S^{1}$ with $\partial M^{3}$ consisting of some tori and capping the boundary components with some copies of $E^{\prime}(n)$ has complexity zero. Among these manifolds we find infinitely many exotic $K 3$ surfaces (Theorem 15.6) and symplectic manifolds with arbitrary fundamental group (Theorem 15.7).

Proof We have $c\left(M \times S^{1}\right)=0$ by Theorem 9.3. The 0 - and 2-handles of $E^{\prime}(n)$ transform into $4-$ and 2-handles when attached to $\partial\left(M \times S^{1}\right)$ : since there is no 3-handle, we have $c(N)=0$.

\subsection{Simply connected manifolds}

We start with the following consequence of Theorem 8.4.

Corollary 15.5 A closed 4-manifold admitting a handle decomposition without 3handles has complexity zero.

Every such manifold is necessarily simply connected. However, it is still unknown whether every simply connected 4-manifold has a handle decomposition without 3-handles (or even without 1- and 3-handles).

For every knot $K \subset S^{3}$, Fintushel and Stern constructed an exotic K3 surface $X_{K}$ whose Seiberg-Witten invariant is roughly the Alexander polynomial of the knot [13]. Among them there are infinitely many distinct exotic K3 surfaces.

Theorem 15.6 The manifold $X_{K}$ has complexity zero for every knot $K$.

Proof The manifold $X_{K}$ is constructed by capping $\left(S^{3} \backslash R(K)\right) \times S^{1}$ with one copy of $E^{\prime}(2)$ [13]. The result follows from Lemma 15.4.

We do not know if $X_{K}$ admits a handle decomposition without 3-handles. 


\subsection{Symplectic manifolds}

Gompf constructed in [15] a family of closed symplectic 4-manifolds with arbitrary fundamental group. It turns out that these manifolds have complexity zero. We can therefore strengthen Corollary 15.2.

Theorem 15.7 Every finitely presented group is the fundamental group of a closed symplectic 4-manifold of complexity zero. The manifold can be chosen to be spin or nonspin.

Proof Gompf's construction starts with a particular 3-manifold $M^{3}$ bounded by some tori, and hence caps the boundary components of $M^{3} \times S^{1}$ with copies of $E^{\prime}(n)$ [15]. The result follows from Lemma 15.4.

\section{References}

[1] E Akin, Manifold phenomena in the theory of polyhedra, Trans. Amer. Math. Soc. 143 (1969) 413-473 MR0253329

[2] S B Alexander, R L Bishop, Spines and homology of thin Riemannian manifolds with boundary, Adv. Math. 155 (2000) 23-48 MR1789847

[3] S B Alexander, R L Bishop, Spines and topology of thin Riemannian manifolds, Trans. Amer. Math. Soc. 355 (2003) 4933-4954 MR1997598

[4] M A Armstrong, Transversality for polyhedra, Ann. of Math. (2) 86 (1967) 172-191 MR0219075

[5] I K Babenko, Asymptotic invariants of smooth manifolds, Izv. Ross. Akad. Nauk Ser. Mat. 56 (1992) 707-751 MR1208148

[6] R Benedetti, C Petronio, Lectures on hyperbolic geometry, Universitext, Springer, Berlin (1992) MR1219310

[7] G Besson, G Courtois, S Gallot, Volume et entropie minimale des espaces localement symétriques, Invent. Math. 103 (1991) 417-445 MR1085114

[8] B A Burton, Enumeration of non-orientable 3-manifolds using face-pairing graphs and union-find, Discrete Comput. Geom. 38 (2007) 527-571 MR2352707

[9] P J Callahan, M V Hildebrand, J R Weeks, A census of cusped hyperbolic 3manifolds, Math. Comp. 68 (1999) 321-332 MR1620219

[10] F Costantino, Complexity of 4-manifolds, Experiment. Math. 15 (2006) 237-249 MR2253547

[11] F Costantino, D Thurston, 3-manifolds efficiently bound 4-manifolds, J. Topol. 1 (2008) 703-745 MR2417451 
[12] S Eilenberg, J A Zilber, Semi-simplicial complexes and singular homology, Ann. of Math. (2) 51 (1950) 499-513 MR0035434

[13] R Fintushel, RJ Stern, Knots, links, and 4-manifolds, Invent. Math. 134 (1998) 363-400 MR1650308

[14] R Frigerio, B Martelli, C Petronio, Small hyperbolic 3-manifolds with geodesic boundary, Experiment. Math. 13 (2004) 171-184 MR2068891

[15] R E Gompf, A new construction of symplectic manifolds, Ann. of Math. (2) 142 (1995) 527-595 MR1356781

[16] R E Gompf, A I Stipsicz, 4-manifolds and Kirby calculus, Graduate Studies in Math. 20, Amer. Math. Soc. (1999) MR1707327

[17] M Gromov, Volume and bounded cohomology, Inst. Hautes Études Sci. Publ. Math. (1982) 5-99 (1983) MR686042

[18] M Gromov, Filling Riemannian manifolds, J. Differential Geom. 18 (1983) 1-147 MR697984

[19] A Hatcher, Algebraic topology, Cambridge Univ. Press (2002) MR1867354

[20] D Kotschick, Entropies, volumes, and Einstein metrics arXiv:math/040215

[21] T Kuessner, Multicomplexes, bounded cohomology and additivity of simplicial volume arXiv:math/0109057

[22] A Manning, Topological entropy for geodesic flows, Ann. of Math. (2) 110 (1979) 567-573 MR554385

[23] B Martelli, Four-manifolds with shadow-complexity zero arXiv:0909.0168

[24] B Martelli, Links, two-handles, and four-manifolds, Int. Math. Res. Not. (2005) 35953623 MR2200121

[25] B Martelli, Complexity of 3-manifolds, from: "Spaces of Kleinian groups", (Y N Minsky, M Sakuma, C Series, editors), London Math. Soc. Lecture Note Ser. 329, Cambridge Univ. Press (2006) 91-120 MR2258746

[26] B Martelli, C Petronio, Three-manifolds having complexity at most 9, Experiment. Math. 10 (2001) 207-236 MR1837672

[27] S V Matveev, Special skeletons of piecewise linear manifolds, Mat. Sb. (N.S.) 92(134) (1973) 282-293, 344 MR0343285

[28] S V Matveev, The theory of the complexity of three-dimensional manifolds, Akad. Nauk Ukrain. SSR Inst. Mat. Preprint (1988) 32 MR982986

[29] S V Matveev, Complexity theory of three-dimensional manifolds, Acta Appl. Math. 19 (1990) 101-130 MR1074221

[30] S V Matveev, Tabulation of three-dimensional manifolds, Uspekhi Mat. Nauk 60 (2005) 97-122 MR2190925 
[31] S V Matveev, Algorithmic topology and classification of 3-manifolds, second edition, Algorithms and Computation in Math. 9, Springer, Berlin (2007) MR2341532

[32] C McCrory, Cone complexes and PL transversality, Trans. Amer. Math. Soc. 207 (1975) 269-291 MR0400243

[33] G Mikhalkin, Decomposition into pairs-of-pants for complex algebraic hypersurfaces, Topology 43 (2004) 1035-1065 MR2079993

[34] H R Morton, Joins of polyhedra, Topology 9 (1970) 243-249 MR0261587

[35] G P Paternain, J Petean, Minimal entropy and collapsing with curvature bounded from below, Invent. Math. 151 (2003) 415-450 MR1953264

[36] C P Rourke, B J Sanderson, Block bundles. I, Ann. of Math. (2) 87 (1968) 1-28 MR0226645

[37] C P Rourke, B J Sanderson, Introduction to piecewise-linear topology, Ergebnisse Math. ihrer Grenzgebiete 69, Springer, New York (1972) MR0350744

[38] D A Stone, Stratified polyhedra, Lecture Notes in Math. 252, Springer, Berlin (1972) MR0372870

[39] J H C Whitehead, On $C^{1}$-complexes, Ann. of Math. (2) 41 (1940) 809-824 MR0002545

Dipartimento di Matematica “Tonelli”, Università di Pisa Largo Pontecorvo 5, 56127 Pisa, Italy

martelli@dm.unipi.it

wWw.dm.unipi.it/ martelli

Received: 5 November $2009 \quad$ Revised: 19 April 2010 